\title{
Composite relaxed extragradient method for triple hierarchical variational inequalities with constraints of systems of variational inequalities
}

\author{
Lu-Chuan Ceng ${ }^{a}$, Yeong-Cheng Liou ${ }^{b, c, *}$, Ching-Feng Wen ${ }^{d}$ \\ a Department of Mathematics, Shanghai Normal University; and Scientific Computing Key Laboratory of Shanghai Universities, \\ Shanghai 200234, China. \\ ${ }^{b}$ Department of Healthcare Administration and Medical Informatics, and Research Center of Nonlinear Analysis and Optimization, \\ Kaohsiung Medical University, Kaohsiung 807, Taiwan. \\ ${ }^{c}$ Department of Medical Research, Kaohsiung Medical University Hospital, Kaohsiung 807, Taiwan. \\ ${ }^{d}$ Center for General Education; and Research Center for Nonlinear Analysis and Optimization, Kaohsiung Medical University, \\ Kaohsiung 807, Taiwan.
}

Communicated by $\mathrm{X}$. Qin

\begin{abstract}
In this paper, we introduce and analyze a composite relaxed extragradient viscosity algorithm for solving the triple hierarchical variational inequality problem with the constraint of general system of variational inequalities in a real Hilbert space. Strong convergence of the iteration sequences generated by the algorithm is established under some suitable conditions. Our results improve and extend the corresponding results in the earlier and recent literature. (c)2017 All rights reserved.
\end{abstract}

Keywords: Composite relaxed extragradient algorithm, triple hierarchical variational inequality, general system of variational inequalities, inverse-strongly monotone mapping.

2010 MSC: 49J30, 47H09, 47J20, 49M05.

\section{Introduction}

Let $\mathrm{H}$ be a real Hilbert space with inner product $\langle\cdot, \cdot\rangle$ and norm $\|\cdot\|$. Let $\mathrm{C}$ be a nonempty closed convex subset of $\mathrm{H}$. Let $\mathrm{S}: \mathrm{C} \rightarrow \mathrm{H}$ be a nonlinear mapping on $\mathrm{C}$. We denote by $\operatorname{Fix}(\mathrm{S})$ the set of fixed points of $S$ and by $R$ the set of all real numbers. A mapping $S: C \rightarrow H$ is called L-Lipschitz continuous if there exists a constant $L \geqslant 0$ such that

$$
\|S x-S y\| \leqslant L\|x-y\|, \quad \forall x, y \in C .
$$

In particular, if $L=1$ then $S$ is called a nonexpansive mapping; if $L \in[0,1)$ then $S$ is called a contraction.

Let $\mathcal{A}: \mathrm{C} \rightarrow \mathrm{H}$ be a nonlinear mapping on $\mathrm{C}$. The classical variational inequality problem (VIP) is to find $x \in \mathrm{C}$ such that

$$
\langle\mathcal{A} x, y-x\rangle \geqslant 0, \quad \forall y \in C .
$$

The solution set of VIP $(1.1)$ is denoted by $\operatorname{VI}(C, \mathcal{A})$.

\footnotetext{
*Corresponding author

Email addresses: zenglc@hotmail.com (Lu-Chuan Ceng), simplex_liou@hotmail.com (Yeong-Cheng Liou), cf wen@kmu . edu. tw (Ching-Feng Wen)

doi:10.22436/jnsa.010.04.58
}

Received 2017-01-10 
We now recall that the metric (or nearest point) projection from $\mathrm{H}$ onto $\mathrm{C}$ is the mapping $\mathrm{P}_{\mathrm{C}}: \mathrm{H} \rightarrow \mathrm{C}$ which assigns to each point $x \in H$ the unique point $P_{C} x \in C$ satisfying the property

$$
\left\|x-P_{C} x\right\|=\inf _{y \in C}\|x-y\|=: d(x, C) .
$$

If $A$ is a strongly monotone and Lipschitz-continuous mapping on $C$, then VIP (1.1) has a unique solution. In order to solve (1.1), Korpelevich [16] proposed the following extragradient algorithm in Euclidean space $\mathbf{R}^{\mathrm{n}}$ :

$$
\left\{\begin{array}{l}
y_{k}=P_{C}\left(x_{k}-\tau A x_{k}\right), \\
x_{k+1}=P_{C}\left(x_{k}-\tau A y_{k}\right), \quad \forall k \geqslant 0 .
\end{array}\right.
$$

The VIP and Korpelevich's extragradient method have received so much attention, see e.g., [2, 6-8, 29, 30] and references therein.

Let $\mathrm{A}: \mathrm{C} \rightarrow \mathrm{H}$ and $\mathrm{B}: \mathrm{H} \rightarrow \mathrm{H}$ be two mappings. Consider the following bilevel variational inequality problem (BVIP).

Problem 1.1. Find $x^{*} \in \mathrm{VI}(\mathrm{C}, \mathrm{B})$ such that

$$
\left\langle A x^{*}, x-x^{*}\right\rangle \geqslant 0, \quad \forall x \in \operatorname{VI}(C, B),
$$

where $\operatorname{VI}(C, B)$ is the set of solutions of the VIP of finding $y^{*} \in C$ such that

$$
\left\langle B y^{*}, y-y^{*}\right\rangle \geqslant 0, \quad \forall y \in C .
$$

Note that Anh et al. [1] studied the above BVIP with $H=\mathbf{R}^{n}$. BVIP includes the classes of mathematical programs with equilibrium constraints ([18]), bilevel minimization problems ([23]), variational inequalities $([3,31,32])$ and complementarity problems as special cases. It is worth pointing out that the BVIP is quite different from other types of variational inequality problems considered in the very recent literature, see e.g., $[9,10,21,22]$.

In what follows, suppose that $A$ and $B$ satisfy the following conditions:

(C1) $B$ is pseudomonotone on $\mathrm{H}$ and $\mathrm{A}$ is $\beta$-strongly monotone on $\mathrm{C}$;

(C2) $A$ is $L_{1}$-Lipschitz continuous on $C$;

(C3) $B$ is $\mathrm{L}_{2}$-Lipschitz continuous on $\mathrm{H}$;

(C4) $\operatorname{VI}(C, B) \neq \emptyset$.

In 2012, Anh et al. [1] introduced the following extragradient iterative algorithm for solving the above bilevel variational inequality.

Algorithm 1.2 ([1]). Initialization. Choose $u \in \mathbf{R}^{n}, x_{0} \in C, 0<\lambda \leqslant \frac{2 \beta}{L_{1}^{2}}$, positive sequences $\left\{\delta_{k}\right\},\left\{\lambda_{k}\right\}$, $\left\{\alpha_{k}\right\},\left\{\beta_{k}\right\},\left\{\gamma_{k}\right\}$, and $\left\{\bar{\epsilon}_{k}\right\}$ such that $\lim _{k \rightarrow \infty} \delta_{k}=0, \sum_{k=0}^{\infty} \bar{\epsilon}_{k}<\infty, \alpha_{k}+\beta_{k}+\gamma_{k}=1 \forall k \geqslant 0, \sum_{k=0}^{\infty} \alpha_{k}=\infty$, $\lim _{k \rightarrow \infty} \alpha_{k}=0, \lim _{k \rightarrow \infty} \beta_{k}=\xi \in\left(0, \frac{1}{2}\right], \lim _{k \rightarrow \infty} \lambda_{k}=0$ and $\lambda_{k} \leqslant \frac{1}{L_{2}}$ for all $k \geqslant 0$.

Step 1. Compute $y_{k}:=P_{C}\left(x_{k}-\lambda_{k} B x_{k}\right)$ and $z_{k}:=P_{C}\left(x_{k}-\lambda_{k} B y_{k}\right)$.

Step 2. Inner loop $j=0,1, \ldots$. Compute

$$
\left\{\begin{array}{l}
x_{k, 0}:=z_{k}-\lambda A z_{k}, \\
y_{k, j}:=P_{C}\left(x_{k, j}-\delta_{j} B x_{k, j}\right), \\
x_{k, j+1}:=\alpha_{j} x_{k, 0}+\beta_{j} x_{k, j}+\gamma_{j} P_{C}\left(x_{k, j}-\delta_{j} B y_{k, j}\right) . \\
\text { If }\left\|x_{k, j+1}-P_{V I(C, B)} x_{k, 0}\right\| \leqslant \bar{\epsilon}_{k} \text { then set } h_{k}:=x_{k, j+1} \text { and go to Step } 3 . \\
\text { Otherwise, increase } j \text { by } 1 \text { and repeat the inner loop Step } 2 .
\end{array}\right.
$$


Step 3. Set $x_{k+1}:=\alpha_{k} u+\beta_{k} x_{k}+\gamma_{k} h_{k}$. Then increase $k$ by 1 and go to Step 1 .

Furthermore, in $[13,14]$, Iiduka introduced the following three-stage variational inequality problem, that is, the following monotone variational inequality with variational inequality constraint over the fixed point set of a nonexpansive mapping.

Problem 1.3 ([14, Problem 3.1]). Assume that

(i) $\mathrm{T}: \mathrm{H} \rightarrow \mathrm{H}$ is a nonexpansive mapping with $\operatorname{Fix}(\mathrm{T}) \neq \emptyset$;

(ii) $A_{1}: H \rightarrow H$ is $\alpha$-inverse strongly monotone;

(iii) $A_{2}: H \rightarrow H$ is $\beta$-strongly monotone and L-Lipschitz continuous;

(iv) $\operatorname{VI}\left(\operatorname{Fix}(T), A_{1}\right) \neq \emptyset$.

Then the objective is to

$$
\text { find } x^{*} \in \operatorname{VI}\left(\operatorname{VI}\left(\operatorname{Fix}(T), A_{1}\right), A_{2}\right):=\left\{x^{*} \in \operatorname{VI}\left(\operatorname{Fix}(T), A_{1}\right):\left\langle A_{2} x^{*}, v-x^{*}\right\rangle \geqslant 0, \forall v \in \operatorname{VI}\left(\operatorname{Fix}(T), A_{1}\right)\right\} \text {. }
$$

Since this problem has a triple structure in contrast with bilevel programming problems $([18,20])$ or hierarchical constrained optimization problems or hierarchical fixed point problem, it is referred to as a triple hierarchical variational inequality problem (THVIP). Very recently, some authors continued the study of Iiduka's THVIP (i.e., Problem 1.3 and its variant and extension; see e.g., [6, 33]).

For solving Problem 1.3, Iiduka presented the following algorithm.

Algorithm 1.4 ([14]). Let $\mathrm{T}: \mathrm{H} \rightarrow \mathrm{H}$ and $A_{i}: H \rightarrow H(i=1,2)$ satisfy the assumptions (i)-(iv) in Problem 1.3 .

Step 0. Take $\left\{\alpha_{k}\right\}_{k=0}^{\infty},\left\{\lambda_{k}\right\}_{k=0}^{\infty} \subset(0, \infty)$, and $\mu>0$, choose $x_{0} \in H$ arbitrarily, and let $k:=0$.

Step 1. Given $x_{k} \in H$, compute $x_{k+1} \in H$ as

$$
\left\{\begin{array}{l}
y_{k}:=T\left(x_{k}-\lambda_{k} A_{1} x_{k}\right), \\
x_{k+1}:=y_{k}-\mu \alpha_{k} A_{2} y_{k} .
\end{array}\right.
$$

Update $k:=k+1$ and go to Step 1 .

On the other hand, let $\mathrm{F}_{1}, \mathrm{~F}_{2}: \mathrm{C} \rightarrow \mathrm{H}$ be two mappings. Consider the following general system of variational inequalities (GSVI) of finding $\left(x^{*}, y^{*}\right) \in C \times C$ such that

$$
\begin{cases}\left\langle v_{1} \mathrm{~F}_{1} \mathrm{y}^{*}+\mathrm{x}^{*}-\mathrm{y}^{*}, x-x^{*}\right\rangle \geqslant 0, & \forall x \in C, \\ \left\langle v_{2} \mathrm{~F}_{2} x^{*}+\mathrm{y}^{*}-x^{*}, y-y^{*}\right\rangle \geqslant 0, & \forall y \in C,\end{cases}
$$

where $v_{1}>0$ and $v_{2}>0$ are two constants. The solution set of GSVI (1.2) is denoted by GSVI $\left(C, F_{1}, F_{2}\right)$. Recently, many authors have been devoting the study of the GSVI (1.2); see e.g., $[3,7,27]$ and the references therein.

In particular, if $F_{1}=F_{2}=\mathcal{A}$, then the GSVI (1.2) reduces to the new system of variational inequalities (NSVI), which was defined by Verma [25]. Further, if $x^{*}=y^{*}$ additionally, then the NSVI reduces to the classical VIP (1.1). In 2008, Ceng et al. [7] transformed the GSVI (1.2) into the fixed point problem of the mapping $G=P_{C}\left(I-v_{1} F_{1}\right) P_{C}\left(I-v_{2} F_{2}\right)$, that is, $G x^{*}=x^{*}$, where $y^{*}=P_{C}\left(I-v_{2} F_{2}\right) x^{*}$. Throughout this paper, the fixed point set of the mapping $\mathrm{G}$ is denoted by GSVI(G).

In 2010, Yao et al. [27] introduced a relaxed extragradient algorithm for finding a common element of the solution set of the GSVI (1.2) and the fixed point set of a strictly pseudocontractive mapping T: C $\rightarrow$, and derived the strong convergence of the proposed algorithm to a common element under some mild conditions.

In this paper, we introduce and analyze a composite relaxed extragradient viscosity algorithm for solving the triple hierarchical variational inequality problem (THVIP) with the constraint of general system of variational inequalities in a real Hilbert space. The proposed algorithm is based on Korpelevich's 
extragradient method [16], Mann's iteration method [2] and composite viscosity approximation method [5]. Under some suitable conditions, the strong convergence of the iteration sequences generated by the algorithm is established. Our results improve and extend the corresponding results announced by some others, e.g., liduka [14], Zeng et al. [33], Anh et al. [1], and Yao et al. [27].

\section{Preliminaries}

Throughout, denoted the weak $\omega$-limit set of the sequence $\left\{x_{k}\right\}$ by $\omega_{w}\left(x_{k}\right)$, i.e.,

$$
\omega_{w}\left(x_{k}\right):=\left\{x \in H: x_{k_{i}} \rightarrow x \text { for some subsequence }\left\{x_{k_{i}}\right\} \text { of }\left\{x_{k}\right\}\right\} .
$$

Definition 2.1. Recall that a mapping $A: C \rightarrow H$ is called

(i) monotone if $\langle A x-A y, x-y\rangle \geqslant 0, \quad \forall x, y \in C$;

(ii) $\eta$-strongly monotone if there exists a constant $\eta>0$ such that $\langle A x-A y, x-y\rangle \geqslant \eta\|x-y\|^{2}, \quad \forall x, y \in$ C;

(iii) $\alpha$-inverse-strongly monotone if there exists a constant $\alpha>0$ such that

$$
\langle A x-A y, x-y\rangle \geqslant \alpha\|A x-A y\|^{2}, \quad \forall x, y \in C .
$$

Some important properties of projections are gathered in the following proposition.

Proposition 2.2 ([26]). For given $\mathrm{x} \in \mathrm{H}$ and $z \in \mathrm{C}$ :

(i) $z=\mathrm{P}_{\mathrm{C}} \mathrm{x} \Leftrightarrow\langle x-z, y-z\rangle \leqslant 0, \forall y \in \mathrm{C}$;

(ii) $z=P_{C} x \Leftrightarrow\|x-z\|^{2} \leqslant\|x-y\|^{2}-\|y-z\|^{2}, \forall y \in C$;

(iii) $\left\langle P_{C} x-P_{C} y, x-y\right\rangle \geqslant\left\|P_{C} x-P_{C} y\right\|^{2}, \forall y \in H$.

Consequently, $\mathrm{P}_{\mathrm{C}}$ is nonexpansive and monotone.

If $A$ is an $\alpha$-inverse-strongly monotone mapping of $C$ into $H$, then it is obvious that $A$ is $\frac{1}{\alpha}$-Lipschitz continuous. We also have that, for all $u, v \in C$ and $\lambda>0$,

$$
\|(I-\lambda A) u-(I-\lambda A) v\|^{2} \leqslant\|u-v\|^{2}+\lambda(\lambda-2 \alpha)\|A u-A v\|^{2} .
$$

So, if $\lambda \leqslant 2 \alpha$, then $\mathrm{I}-\lambda \mathrm{A}$ is a nonexpansive mapping from $\mathrm{C}$ to $\mathrm{H}$.

Definition 2.3. A mapping $\mathrm{T}: \mathrm{H} \rightarrow \mathrm{H}$ is said to be:

(a) nonexpansive if $\|\mathrm{T} x-\mathrm{Ty}\| \leqslant\|x-y\|, \quad \forall x, y \in \mathrm{H}$;

(b) firmly nonexpansive if $2 \mathrm{~T}-\mathrm{I}$ is nonexpansive, or equivalently, if $\mathrm{T}$ is 1-inverse strongly monotone (1-ism), $\langle x-y, T x-T y\rangle \geqslant\|T x-T y\|^{2}, \quad \forall x, y \in H$; alternatively, $T$ is firmly nonexpansive if and only if $\mathrm{T}$ can be expressed as $\mathrm{T}=\frac{1}{2}(\mathrm{I}+\mathrm{S})$, where $\mathrm{S}: \mathrm{H} \rightarrow \mathrm{H}$ is nonexpansive; projections are firmly nonexpansive.

It can be easily seen that if $\mathrm{T}$ is nonexpansive, then $\mathrm{I}-\mathrm{T}$ is monotone. It is also easy to see that a projection $\mathrm{P}_{\mathrm{C}}$ is 1-ism. Inverse strongly monotone (also referred to as co-coercive) operators have been applied widely in solving practical problems in various fields.

Proposition 2.4 ([12]). Let $\mathrm{T}: \mathrm{C} \rightarrow \mathrm{C}$ be a nonexpansive mapping. Then the followings hold:

(i) $\operatorname{Fix}(\mathrm{T})$ is closed and convex;

(ii) $\operatorname{Fix}(\mathrm{T}) \neq \emptyset$ when $\mathrm{C}$ is bounded.

We need some facts and tools in a real Hilbert space $\mathrm{H}$ which are listed as lemmas below. 
Lemma 2.5. Let $\mathrm{X}$ be a real inner product space. Then there holds the following inequality

$$
\|x+y\|^{2} \leqslant\|x\|^{2}+2\langle y, x+y\rangle, \quad \forall x, y \in X .
$$

Recall that, a mapping $\mathrm{A}: \mathrm{C} \rightarrow \mathrm{H}$ is called hemicontinuous if for all $\mathrm{x}, \mathrm{y} \in \mathrm{C}$, the mapping $\mathrm{g}:[0,1] \rightarrow$ $H$, defined by $g(t):=A(t x+(1-t) y)$, is continuous. Some properties of the solution set of the monotone variational inequality are mentioned in the following result.

Lemma $2.6([15,24])$. Let $\mathrm{A}: \mathrm{C} \rightarrow \mathrm{H}$ be a monotone and hemicontinuous mapping. Then the following hold:

(i) $\operatorname{VI}(C, A)$ is equivalent to $\operatorname{MVI}(C, A):=\left\{x^{*} \in C:\left\langle A y, y-x^{*}\right\rangle \geqslant 0, \forall y \in C\right\}$;

(ii) $\operatorname{VI}(C, A) \neq \emptyset$ when $C$ is bounded;

(iii) $\operatorname{VI}(C, A)=\operatorname{Fix}\left(\mathrm{P}_{C}(\mathrm{I}-\lambda \mathrm{A})\right)$ for all $\lambda>0$, where $\mathrm{I}$ is the identity mapping on $\mathrm{H}$;

(iv) $\operatorname{VI}(C, A)$ consists of only one point, if $A$ is strongly monotone and Lipschitz continuous.

Lemma 2.7 ([11]). Let $\mathrm{C}$ be a nonempty closed convex subset of a real Hilbert space $\mathrm{H}$. Let $\mathrm{S}$ be a nonexpansive self-mapping on $\mathrm{C}$ with $\operatorname{Fix}(\mathrm{S}) \neq \emptyset$. Then $\mathrm{I}-\mathrm{S}$ is demiclosed. That is, whenever $\left\{\mathrm{x}_{\mathrm{k}}\right\}$ is a sequence in $\mathrm{C}$ weakly converging to some $\mathrm{x} \in \mathrm{C}$ and the sequence $\left\{(\mathrm{I}-\mathrm{S}) \mathrm{x}_{\mathrm{k}}\right\}$ strongly converges to some $\mathrm{y}$, it follows that $(\mathrm{I}-\mathrm{S}) \mathrm{x}=\mathrm{y}$. Here $\mathrm{I}$ is the identity operator of $\mathrm{H}$.

Recall that, a mapping $\mathrm{T}: \mathrm{C} \rightarrow \mathrm{C}$ is called a $\zeta$-strictly pseudocontractive mapping (or a $\zeta$-strict pseudocontraction) if there exists a constant $\zeta \in[0,1)$ such that

$$
\|\mathrm{T} x-\mathrm{T} y\|^{2} \leqslant\|x-y\|^{2}+\zeta\|(\mathrm{I}-\mathrm{T}) x-(\mathrm{I}-\mathrm{T}) y\|^{2}, \quad \forall x, y \in \mathrm{C} .
$$

Lemma 2.8 ([19]). Let $\mathrm{C}$ be a nonempty closed convex subset of a real Hilbert space $\mathrm{H}$ and $\mathrm{T}: \mathrm{C} \rightarrow \mathrm{C}$ be a mapping.

(i) If $\mathrm{T}$ is a $\zeta$-strictly pseudocontractive mapping, then $\mathrm{T}$ satisfies the Lipschitzian condition

$$
\|\mathrm{T} x-\mathrm{T} y\| \leqslant \frac{1+\zeta}{1-\zeta}\|x-y\|, \quad \forall x, y \in C .
$$

(ii) If $\mathrm{T}$ is a $\zeta$-strictly pseudocontractive mapping, then the mapping $\mathrm{I}-\mathrm{T}$ is semiclosed at 0 , that is, if $\left\{\mathrm{x}_{\mathrm{n}}\right\}$ is a sequence in $\mathrm{C}$ such that $\mathrm{x}_{\mathrm{n}} \rightarrow \tilde{\mathrm{x}}$ and $(\mathrm{I}-\mathrm{T}) \mathrm{x}_{\mathrm{n}} \rightarrow 0$, then $(\mathrm{I}-\mathrm{T}) \tilde{\mathrm{x}}=0$.

(iii) If $\mathrm{T}$ is $\zeta$-(quasi-)strict pseudocontraction, then the fixed-point set $\operatorname{Fix}(\mathrm{T})$ of $\mathrm{T}$ is closed and convex so that the projection $\mathrm{P}_{\mathrm{Fix}(\mathrm{T})}$ is well-defined.

Lemma 2.9 ([27]). Let $\mathrm{C}$ be a nonempty closed convex subset of a real Hilbert space $\mathrm{H}$. Let $\mathrm{T}: \mathrm{C} \rightarrow \mathrm{C}$ be a $\zeta$-strictly pseudocontractive mapping. Let $\gamma$ and $\delta$ be two nonnegative real numbers such that $(\gamma+\delta) \zeta \leqslant \gamma$. Then

$$
\|\gamma(x-y)+\delta(T x-T y)\| \leqslant(\gamma+\delta)\|x-y\|, \quad \forall x, y \in C .
$$

Lemma 2.10. Let $\mathrm{f}: \mathrm{C} \rightarrow \mathrm{C}$ be a $\rho$-contraction with $\rho \in[0,1)$. Then $\mathrm{I}-\mathrm{f}$ is $(1-\rho)$-strongly monotone, that is,

$$
\langle(I-f) x-(I-f) y, x-y\rangle \geqslant(1-\rho)\|x-y\|^{2}, \quad \forall x, y \in C .
$$

Lemma 2.11 ([7]). For given $x^{*}, y^{*} \in C,\left(x^{*}, y^{*}\right)$ is a solution of the GSVI (1.2) if and only if $x^{*}$ is a fixed point of the mapping $\mathrm{G}: \mathrm{C} \rightarrow \mathrm{C}$ defined by

$$
\mathrm{G} x=\mathrm{P}_{\mathrm{C}}\left(\mathrm{I}-v_{1} \mathrm{~F}_{1}\right) \mathrm{P}_{\mathrm{C}}\left(\mathrm{I}-v_{2} \mathrm{~F}_{2}\right) x, \quad \forall x \in \mathrm{C},
$$

where $\mathrm{y}^{*}=\mathrm{P}_{\mathrm{C}}\left(\mathrm{I}-\mathrm{v}_{2} \mathrm{~F}_{2}\right) \mathrm{x}^{*}$.

In particular, if the mapping $F_{i}: C \rightarrow H$ is $\zeta_{i}$-inverse-strongly monotone for $i=1,2$, then the mapping $G$ is nonexpansive provided $v_{i} \in\left(0,2 \zeta_{i}\right]$ for $i=1,2$. We denote by GSVI $(G)$ the fixed point set of the mapping G. 
Lemma 2.12 ([17]). Let $\left\{a_{k}\right\}$ be a sequence of nonnegative real numbers satisfying the property

$$
a_{k+1} \leqslant\left(1-s_{k}\right) a_{k}+s_{k} t_{k}+r_{k}, \quad \forall k \geqslant 0,
$$

where $\left\{s_{k}\right\},\left\{t_{k}\right\}$, and $\left\{r_{k}\right\}$ are sequences of real numbers such that

(i) $\left\{s_{k}\right\} \subset[0,1]$ and $\sum_{k=0}^{\infty} s_{k}=\infty$;

(ii) either $\lim \sup _{k \rightarrow \infty} t_{k} \leqslant 0$, or $\sum_{k=0}^{\infty}\left|s_{k} t_{k}\right|<\infty$;

(iii) $\sum_{k=0}^{\infty} r_{k}<\infty$ with $r_{k} \geqslant 0, \forall k \geqslant 0$.

Then, $\lim _{\mathrm{k} \rightarrow \infty} \mathrm{a}_{\mathrm{k}}=0$.

Lemma 2.13 ([11]). Let $\mathrm{H}$ be a real Hilbert space. Then the followings hold:

(a) $\|x-y\|^{2}=\|x\|^{2}-\|y\|^{2}-2\langle x-y, y\rangle$ for all $x, y \in H$;

(b) $\|\lambda x+\mu y\|^{2}=\lambda\|x\|^{2}+\mu\|y\|^{2}-\lambda \mu\|x-y\|^{2}$ for all $x, y \in H$ and $\lambda, \mu \in[0,1]$ with $\lambda+\mu=1$;

(c) if $\left\{x_{k}\right\}$ is a sequence in $\mathrm{H}$ such that $\mathrm{x}_{\mathrm{k}} \rightarrow \mathrm{x}$, it follows that

$$
\limsup _{k \rightarrow \infty}\left\|x_{k}-y\right\|^{2}=\limsup _{k \rightarrow \infty}\left\|x_{k}-x\right\|^{2}+\|x-y\|^{2}, \quad \forall y \in H .
$$

Lemma 2.14 ([4]). Let $\left\{a_{k}\right\}_{k=0}^{\infty}$ be a bounded sequence of nonnegative real numbers and $\left\{b_{k}\right\}_{k=0}^{\infty}$ be a sequence of real numbers such that $\limsup _{k \rightarrow \infty} b_{k} \leqslant 0$. Then, $\limsup _{k \rightarrow \infty} a_{k} b_{k} \leqslant 0$.

\section{Main results}

Let $\mathrm{H}$ be a real Hilbert space. In this section, we always assume the followings.

- $\mathrm{F}_{\mathrm{i}}: \mathrm{H} \rightarrow \mathrm{H}$ is $\zeta_{i}$-inverse strongly monotone for $i=1,2$ and $\mathrm{T}: \mathrm{H} \rightarrow \mathrm{H}$ is a $\zeta$-strictly pseudocontractive mapping;

- $\mathrm{G}: \mathrm{H} \rightarrow \mathrm{C}$ is a mapping defined by $\mathrm{G} x=\mathrm{P}_{\mathrm{C}}\left(\mathrm{I}-v_{1} \mathrm{~F}_{1}\right) \mathrm{P}_{\mathrm{C}}\left(\mathrm{I}-v_{2} \mathrm{~F}_{2}\right) x$ with $0<v_{i}<2 \zeta_{i}$ for $i=1,2$;

- $\mathrm{f}: \mathrm{H} \rightarrow \mathrm{H}$ is a $\rho$-contraction mapping with $\rho \in[0,1)$;

- $\mathrm{A}: \mathrm{H} \rightarrow \mathrm{H}$ and $\mathrm{B}: \mathrm{H} \rightarrow \mathrm{H}$ are two mappings such that the hypotheses (H1)-(H4) hold:

(H1) B is monotone on $\mathrm{H}$,

(H2) $A$ is $\beta$-inverse-strongly monotone on $\mathrm{H}$,

(H3) $B$ is L-Lipschitz continuous on $\mathrm{H}$,

(H4) $\Omega:=\operatorname{VI}(\operatorname{VI}(\operatorname{GSVI}(\mathrm{G}) \cap \operatorname{Fix}(\mathrm{T}), \mathrm{B}), \mathrm{A}) \neq \emptyset$.

Next, we introduce the following triple hierarchical variational inequality problem (THVIP) defined over the common solution set of the GSVI (1.2) and the fixed point problem of a strictly pseudocontractive mapping $\mathrm{T}$.

Problem 3.1. The objective is to

$$
\text { find } \begin{aligned}
x^{*} \in \Omega & :=\operatorname{VI}(\operatorname{VI}(\operatorname{GSVI}(G) \cap \operatorname{Fix}(T), B), A) \\
& :=\left\{x^{*} \in \operatorname{VI}(\operatorname{GSVI}(G) \cap \operatorname{Fix}(T), B):\left\langle A x^{*}, x-x^{*}\right\rangle \geqslant 0, \forall x \in \operatorname{VI}(\operatorname{GSVI}(G) \cap \operatorname{Fix}(T), B)\right\} .
\end{aligned}
$$

That is, the $\Omega$ is the solution set of the THVIP of finding $x^{*} \in \operatorname{VI}(\operatorname{GSVI}(G) \cap \operatorname{Fix}(T), B)$ such that

$$
\left\langle A x^{*}, x-x^{*}\right\rangle \geqslant 0, \quad \forall x \in \operatorname{VI}(\operatorname{GSVI}(G) \cap \operatorname{Fix}(T), B),
$$

where $\operatorname{VI}(G S V I(G) \cap \operatorname{Fix}(T), B)$ denotes the set of solutions of the VIP of finding $y^{*} \in \operatorname{GSVI}(G) \cap \operatorname{Fix}(T)$ such that

$$
\left\langle\mathrm{By}^{*}, \mathrm{y}-\mathrm{y}^{*}\right\rangle \geqslant 0, \quad \forall \mathrm{y} \in \mathrm{GSVI}(\mathrm{G}) \cap \operatorname{Fix}(\mathrm{T}) .
$$

It is worth pointing out that Problem 3.1 is very different from Problem 1.3 because the solution set of Problem 3.1 may not be a singleton but the solution set of Problem 1.3 must be a singleton. 
Algorithm 3.2. Choose $u \in H, x_{0} \in H, k=0,0<\lambda \leqslant 2 \beta$, positive sequences $\left\{\delta_{k}\right\},\left\{\lambda_{k}\right\},\left\{\alpha_{k}\right\},\left\{\beta_{k}\right\},\left\{\gamma_{k}\right\}$, and $\left\{\bar{\epsilon}_{k}\right\}$ such that $\lim _{k \rightarrow \infty} \delta_{k}=0, \sum_{k=0}^{\infty} \bar{\epsilon}_{k}<\infty, \alpha_{k}+\beta_{k}+\gamma_{k}=1, \sum_{k=0}^{\infty} \alpha_{k}=\infty, \lim _{k \rightarrow \infty} \alpha_{k}=0, \lim _{k \rightarrow \infty} \beta_{k}=\xi \in\left(\zeta, \frac{1}{2}\right]$, $\lim _{k \rightarrow \infty} \lambda_{k}=0$, and $\lambda_{k} \leqslant \frac{1}{L}$ for all $k \geqslant 0$.

Step 1. Compute

$$
\left\{\begin{array}{l}
u_{k}:=\alpha_{k} f\left(x_{k}\right)+\left(1-\alpha_{k}\right) G x_{k}, \\
v_{k}:=\alpha_{k} u_{k}+\beta_{k} x_{k}+\gamma_{k}\left[\mu G x_{k}+(1-\mu) T G x_{k}\right], \\
y_{k}:=P_{G S V I(G) \cap F i x(T)}\left(v_{k}-\lambda_{k} B v_{k}\right), \\
z_{k}:=P_{G S V I(G) \cap \operatorname{Fix}(T)}\left(v_{k}-\lambda_{k} B y_{k}\right) .
\end{array}\right.
$$

Step 2. Inner loop $j=0,1, \ldots$. Compute

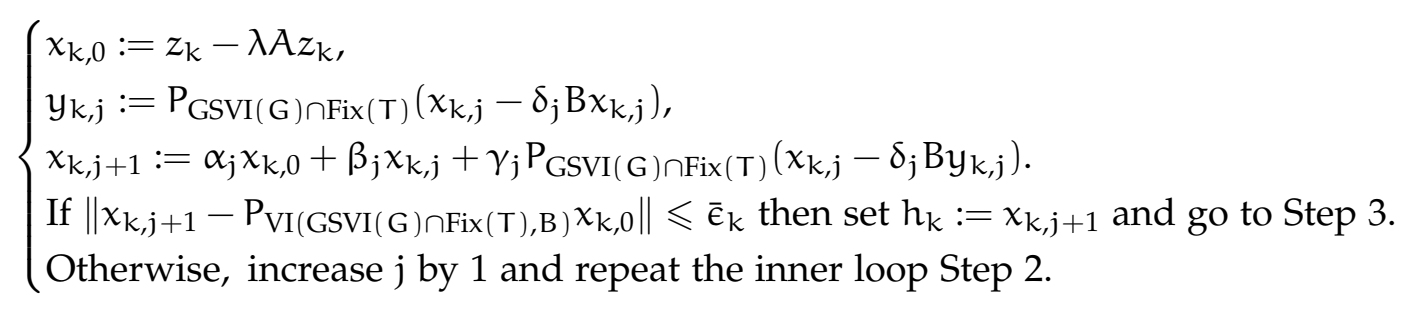

Step 3. Set $x_{k+1}:=\alpha_{k} u+\beta_{k} x_{k}+\gamma_{k} h_{k}$. Then increase $k$ by 1 and go to Step 1 .

Let $\mathrm{C}$ be a nonempty closed convex subset of $\mathrm{H}, \mathrm{B}: \mathrm{C} \rightarrow \mathrm{H}$ be monotone and L-Lipschitz continuous on $C$, and $S: C \rightarrow C$ be a nonexpansive mapping such that $\operatorname{VI}(C, B) \cap \operatorname{Fix}(S) \neq \emptyset$. Let the sequences $\left\{x_{n}\right\}$ and $\left\{y_{n}\right\}$ be generated by

$$
\left\{\begin{array}{l}
x_{0} \in C \text { chosen arbitrarily, } \\
y_{k}=P_{C}\left(x_{k}-\delta_{k} B x_{k}\right), \\
x_{k+1}=\alpha_{k} x_{0}+\beta_{k} x_{k}+\gamma_{k} S P_{C}\left(x_{k}-\delta_{k} B y_{k}\right), \quad \forall k \geqslant 0
\end{array}\right.
$$

where $\left\{\alpha_{k}\right\},\left\{\beta_{k}\right\},\left\{\gamma_{k}\right\}$, and $\left\{\delta_{k}\right\}$ satisfy the following conditions: $\delta_{k}>0, \lim _{k \rightarrow \infty} \delta_{k}=0, \alpha_{k}+\beta_{k}+\gamma_{k}=1$, $\sum_{k=0}^{\infty} \alpha_{k}=\infty, \lim _{k \rightarrow \infty} \alpha_{k}=0$, and $0<\liminf _{k \rightarrow \infty} \beta_{k} \leqslant \limsup _{k \rightarrow \infty} \beta_{k}<1$ for all $k \geqslant 0$. Under these conditions, Yao et al. [28] proved that the sequences $\left\{x_{k}\right\}$ and $\left\{y_{k}\right\}$ converge strongly to the same point $P_{V I(C, B) \cap F i x(S)} x_{0}$.

Applying these iteration sequences with $S$ being the identity mapping, we have the following lemma.

Lemma 3.3. Suppose that the hypotheses (H1)-(H4) hold. Then the sequence $\left\{x_{k, j}\right\}$ generated by Algorithm 3.2

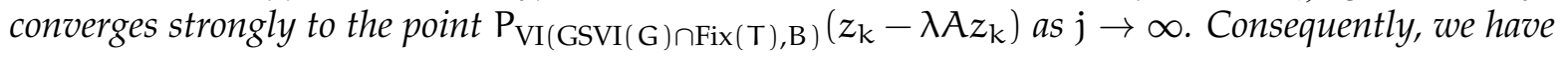

$$
\left\|h_{k}-P_{\operatorname{VI}(\operatorname{GSVI}(G) \cap \operatorname{Fix}(T), B)}\left(z_{k}-\lambda A z_{k}\right)\right\| \leqslant \bar{\epsilon}_{k}, \forall k \geqslant 0 .
$$

In the sequel, we always suppose that the inner loop in Algorithm 3.2 terminates after a finite number of steps. This assumption, by Lemma 3.3, is satisfied when B is monotone on GSVI $(G) \cap \operatorname{Fix}(T)$.

Lemma 3.4. Let the sequences $\left\{v_{\mathrm{k}}\right\},\left\{\mathrm{y}_{\mathrm{k}}\right\}$, and $\left\{z_{\mathrm{k}}\right\}$ be generated by Algorithm 3.2, B be L-Lipschitzian and monotone on $\mathrm{H}$, and $\mathrm{p} \in \operatorname{VI}(\mathrm{GSVI}(\mathrm{G}) \cap \operatorname{Fix}(\mathrm{T}), \mathrm{B})$. Then, we have

$$
\left\|z_{k}-p\right\|^{2} \leqslant\left\|v_{k}-p\right\|^{2}-\left(1-\lambda_{k} L\right)\left\|v_{k}-y_{k}\right\|^{2}-\left(1-\lambda_{k} L\right)\left\|y_{k}-z_{k}\right\|^{2} .
$$

Proof. Let $p \in \operatorname{VI}(\mathrm{GSVI}(\mathrm{G}) \cap \operatorname{Fix}(\mathrm{T})$, B $)$. That means

$$
\langle\mathrm{Bp}, x-\mathrm{p}\rangle \geqslant 0, \quad \forall x \in \mathrm{GSVI}(\mathrm{G}) \cap \operatorname{Fix}(\mathrm{T}) .
$$


Then, for each $\lambda_{k}>0, p$ satisfies the fixed point equation $p=P_{G S V I(G) \cap F i x(T)}\left(p-\lambda_{k} B p\right)$. Since $B$ is monotone on $\mathrm{H}$ and $\mathrm{p} \in \mathrm{VI}(\mathrm{GSVI}(\mathrm{G}) \cap \operatorname{Fix}(\mathrm{T})$, B $)$, we have

$$
\left\langle B y_{k}, y_{k}-p\right\rangle \geqslant\left\langle B p, y_{k}-p\right\rangle \geqslant 0 \text {. }
$$

Then, applying Proposition 2.2 (ii) with $v_{k}-\lambda_{k} B y_{k}$ and $p$, we obtain

$$
\begin{aligned}
\left\|z_{k}-p\right\|^{2} \leqslant & \left\|v_{k}-\lambda_{k} B y_{k}-p\right\|^{2}-\left\|v_{k}-\lambda_{k} B y_{k}-z_{k}\right\|^{2} \\
= & \left\|v_{k}-p\right\|^{2}-2 \lambda_{k}\left\langle B y_{k}, v_{k}-p\right\rangle+\lambda_{k}^{2}\left\|B y_{k}\right\|^{2}-\left\|v_{k}-z_{k}\right\|^{2} \\
& -\lambda_{k}^{2}\left\|B y_{k}\right\|^{2}+2 \lambda_{k}\left\langle B y_{k}, v_{k}-z_{k}\right\rangle \\
= & \left\|v_{k}-p\right\|^{2}-\left\|v_{k}-z_{k}\right\|^{2}+2 \lambda_{k}\left\langle B y_{k}, p-z_{k}\right\rangle \\
= & \left\|v_{k}-p\right\|^{2}-\left\|v_{k}-z_{k}\right\|^{2}+2 \lambda_{k}\left\langle B y_{k}, p-y_{k}\right\rangle+2 \lambda_{k}\left\langle B y_{k}, y_{k}-z_{k}\right\rangle \\
\leqslant & \left\|v_{k}-p\right\|^{2}-\left\|v_{k}-z_{k}\right\|^{2}+2 \lambda_{k}\left\langle B y_{k}, y_{k}-z_{k}\right\rangle .
\end{aligned}
$$

Applying Proposition 2.2 (i) with $v_{k}-\lambda_{k} B v_{k}$ and $z_{k}$, we also have

$$
\left\langle v_{k}-\lambda_{k} B v_{k}-y_{k}, z_{k}-y_{k}\right\rangle \leqslant 0 .
$$

Combining this inequality with (3.3) and observing that B is L-Lipschitz continuous on $\mathrm{H}$, we obtain

$$
\begin{aligned}
\left\|z_{k}-p\right\|^{2} \leqslant & \left\|v_{k}-p\right\|^{2}-\left\|\left(v_{k}-y_{k}\right)+\left(y_{k}-z_{k}\right)\right\|^{2}+2 \lambda_{k}\left\langle B y_{k}, y_{k}-z_{k}\right\rangle \\
= & \left\|v_{k}-p\right\|^{2}-\left\|v_{k}-y_{k}\right\|^{2}-\left\|y_{k}-z_{k}\right\|^{2}-2\left\langle v_{k}-y_{k}, y_{k}-z_{k}\right\rangle+2 \lambda_{k}\left\langle B y_{k}, y_{k}-z_{k}\right\rangle \\
= & \left\|v_{k}-p\right\|^{2}-\left\|v_{k}-y_{k}\right\|^{2}-\left\|y_{k}-z_{k}\right\|^{2}-2\left\langle v_{k}-\lambda_{k} B y_{k}-y_{k}, y_{k}-z_{k}\right\rangle \\
= & \left\|v_{k}-p\right\|^{2}-\left\|v_{k}-y_{k}\right\|^{2}-\left\|y_{k}-z_{k}\right\|^{2}-2\left\langle v_{k}-\lambda_{k} B v_{k}-y_{k}, y_{k}-z_{k}\right\rangle \\
& +2 \lambda_{k}\left\langle B v_{k}-B y_{k}, z_{k}-y_{k}\right\rangle \\
\leqslant & \left\|v_{k}-p\right\|^{2}-\left\|v_{k}-y_{k}\right\|^{2}-\left\|y_{k}-z_{k}\right\|^{2}+2 \lambda_{k}\left\langle B v_{k}-B y_{k}, z_{k}-y_{k}\right\rangle \\
\leqslant & \left\|v_{k}-p\right\|^{2}-\left\|v_{k}-y_{k}\right\|^{2}-\left\|y_{k}-z_{k}\right\|^{2}+2 \lambda_{k}\left\|B v_{k}-B y_{k}\right\|\left\|z_{k}-y_{k}\right\| \\
\leqslant & \left\|v_{k}-p\right\|^{2}-\left\|v_{k}-y_{k}\right\|^{2}-\left\|y_{k}-z_{k}\right\|^{2}+2 \lambda_{k} L\left\|v_{k}-y_{k}\right\|\left\|z_{k}-y_{k}\right\| \\
\leqslant & \left\|v_{k}-p\right\|^{2}-\left\|v_{k}-y_{k}\right\|^{2}-\left\|y_{k}-z_{k}\right\|^{2}+\lambda_{k} L\left(\left\|v_{k}-y_{k}\right\|^{2}+\left\|z_{k}-y_{k}\right\|^{2}\right) \\
\leqslant & \left\|v_{k}-p\right\|^{2}-\left(1-\lambda_{k} L\right)\left\|v_{k}-y_{k}\right\|^{2}-\left(1-\lambda_{k} L\right)\left\|y_{k}-z_{k}\right\|^{2} .
\end{aligned}
$$

Lemma 3.5. Suppose that the hypotheses $(\mathrm{H} 1)-(\mathrm{H} 4)$ hold. Then the sequence $\left\{x_{\mathrm{k}}\right\}$ generated by Algorithm 3.2 is bounded.

Proof. Since $\lim _{k \rightarrow \infty} \alpha_{k}=0, \lim _{k \rightarrow \infty} \beta_{k}=\xi \in\left(\zeta, \frac{1}{2}\right]$ and $\alpha_{k}+\beta_{k}+\gamma_{k}=1$, we get $\lim _{k \rightarrow \infty}\left(1-\gamma_{k}\right)=$ $\lim _{k \rightarrow \infty}\left(\alpha_{k}+\beta_{k}\right)=\xi$. Moreover, we may assume, without loss of generality, that $\left\{\beta_{k}\right\} \subset[a, b] \subset(\zeta, 1)$. Take an arbitrary $p \in \Omega:=\operatorname{VI}(\operatorname{VI}(\operatorname{GSVI}(G) \cap \operatorname{Fix}(\mathrm{T}), \mathrm{B}), \mathrm{A})$. Putting $\sigma=1-\mu$ and $\mathcal{A}=\mathrm{I}-\mathrm{T}$, we know that $\mathcal{A}$ is $\frac{1-\zeta}{2}$-inverse-strongly monotone since $T$ is $\zeta$-strictly pseudocontractive. We write $\tilde{u}_{k}=\mu G x_{k}+$ $(1-\mu) \mathrm{TG} x_{k}$ for $k \geqslant 0$. Then we observe that $\tilde{u}_{k}=\mu G x_{k}+(1-\mu) T G x_{k}=G x_{k}-(1-\mu)(I-T) G x_{k}=$ $G x_{k}-\sigma \mathcal{A} G x_{k}$, which together with (2.1), yields

$$
\begin{aligned}
\left\|\tilde{u}_{k}-p\right\|^{2} & =\left\|G x_{k}-\sigma \mathcal{A} G x_{k}-(p-\sigma \mathcal{A p})\right\|^{2} \\
& =\left\|G x_{k}-p-\sigma\left(\mathcal{A G} x_{k}-\mathcal{A} p\right)\right\|^{2} \\
& \leqslant\left\|G x_{k}-p\right\|^{2}-\sigma(1-\zeta-\sigma)\left\|\mathcal{A G} x_{k}-\mathcal{A p}\right\|^{2} \\
& =\left\|G x_{k}-p\right\|^{2}-(1-\mu)(\mu-\zeta)\left\|G x_{k}-T G x_{k}\right\|^{2} \\
& \leqslant\left\|G x_{k}-p\right\|^{2} .
\end{aligned}
$$

Since $p=G p=P_{C}\left(I-v_{1} F_{1}\right) P_{C}\left(I-v_{2} F_{2}\right) p$ and $F_{i}$ is $\zeta_{i}$-inverse-strongly monotone with $0<v_{i}<2 \zeta_{i}$ for $i=1,2$, we deduce that 


$$
\begin{aligned}
\left\|G x_{k}-p\right\|^{2}= & \left\|P_{C}\left(I-v_{1} F_{1}\right) P_{C}\left(I-v_{2} F_{2}\right) x_{k}-P_{C}\left(I-v_{1} F_{1}\right) P_{C}\left(I-v_{2} F_{2}\right) p\right\|^{2} \\
\leqslant & \left\|\left(I-v_{1} F_{1}\right) P_{C}\left(I-v_{2} F_{2}\right) x_{k}-\left(I-v_{1} F_{1}\right) P_{C}\left(I-v_{2} F_{2}\right) p\right\|^{2} \\
= & \left\|\left[P_{C}\left(I-v_{2} F_{2}\right) x_{k}-P_{C}\left(I-v_{2} F_{2}\right) p\right]-v_{1}\left[F_{1} P_{C}\left(I-v_{2} F_{2}\right) x_{k}-F_{1} P_{C}\left(I-v_{2} F_{2}\right) p\right]\right\|^{2} \\
\leqslant & \left\|P_{C}\left(I-v_{2} F_{2}\right) x_{k}-P_{C}\left(I-v_{2} F_{2}\right) p\right\|^{2} \\
& +v_{1}\left(v_{1}-2 \zeta_{1}\right)\left\|F_{1} P_{C}\left(I-v_{2} F_{2}\right) x_{k}-F_{1} P_{C}\left(I-v_{2} F_{2}\right) p\right\|^{2} \\
\leqslant & \left\|P_{C}\left(I-v_{2} F_{2}\right) x_{k}-P_{C}\left(I-v_{2} F_{2}\right) p\right\|^{2} \\
\leqslant & \left\|\left(I-v_{2} F_{2}\right) x_{k}-\left(I-v_{2} F_{2}\right) p\right\|^{2} \\
= & \left\|\left(x_{k}-p\right)-v_{2}\left(F_{2} x_{k}-F_{2} p\right)\right\|^{2} \\
\leqslant & \left\|x_{k}-p\right\|^{2}+v_{2}\left(v_{2}-2 \zeta_{2}\right)\left\|F_{2} x_{k}-F_{2} p\right\|^{2} \\
\leqslant & \left\|x_{k}-p\right\|^{2} .
\end{aligned}
$$

So, it follows that

$$
\begin{aligned}
\left\|u_{k}-p\right\| & =\left\|\alpha_{k}\left(f\left(x_{k}\right)-f(p)\right)+\left(1-\alpha_{k}\right)\left(G x_{k}-p\right)+\alpha_{k}(f(p)-p)\right\| \\
& \leqslant \alpha_{k}\left\|f\left(x_{k}\right)-f(p)\right\|+\left(1-\alpha_{k}\right)\left\|G x_{k}-p\right\|+\alpha_{k}\|f(p)-p\| \\
& \leqslant \alpha_{k} \rho\left\|x_{k}-p\right\|+\left(1-\alpha_{k}\right)\left\|x_{k}-p\right\|+\alpha_{k}\|f(p)-p\| \\
& =\left(1-\alpha_{k}(1-\rho)\right)\left\|x_{k}-p\right\|+\alpha_{k}\|f(p)-p\| \\
& =\left(1-\alpha_{k}(1-\rho)\right)\left\|x_{k}-p\right\|+\alpha_{k}(1-\rho) \frac{\|f(p)-p\|}{1-\rho} \\
& \leqslant \max \left\{\left\|x_{k}-p\right\|, \frac{\|f(p)-p\|}{1-\rho}\right\} .
\end{aligned}
$$

Thus, from (3.1) and (3.5), (3.6), and (3.7) we get

$$
\begin{aligned}
\left\|v_{k}-p\right\| & =\left\|\alpha_{k}\left(u_{k}-p\right)+\beta_{k}\left(x_{k}-p\right)+\gamma_{k}\left[\mu G x_{k}+(1-\mu) T G x_{k}-p\right]\right\| \\
& =\left\|\alpha_{k}\left(u_{k}-p\right)+\beta_{k}\left(x_{k}-p\right)+\gamma_{k}\left(\tilde{u}_{k}-p\right)\right\| \\
& \leqslant \alpha_{k}\left\|u_{k}-p\right\|+\beta_{k}\left\|x_{k}-p\right\|+\gamma_{k}\left\|\tilde{u}_{k}-p\right\| \\
& \leqslant \alpha_{k} \max \left\{\left\|x_{k}-p\right\|, \frac{\|f(p)-p\|_{1}}{1-\rho}\right\}+\beta_{k}\left\|x_{k}-p\right\|+\gamma_{k}\left\|G x_{k}-p\right\| \\
& \leqslant \alpha_{k} \max \left\{\left\|x_{k}-p\right\|, \frac{\|f(p)-p\|_{1}}{1-\rho}\right\}+\beta_{k}\left\|x_{k}-p\right\|+\gamma_{k}\left\|x_{k}-p\right\| \\
& =\alpha_{k} \max \left\{\left\|x_{k}-p\right\|, \frac{\|f(p)-p\|}{1-\rho}\right\}+\left(1-\alpha_{k}\right)\left\|x_{k}-p\right\| \\
& \leqslant \max \left\{\left\|x_{k}-p\right\|, \frac{\|f(p)-p\|}{1-\rho}\right\} .
\end{aligned}
$$

On the other hand, for $p \in \Omega$, we have

$$
\langle A p, x-p\rangle \geqslant 0, \quad \forall x \in \operatorname{VI}(\operatorname{GSVI}(G) \cap \operatorname{Fix}(T), B),
$$

which implies $p=P_{\mathrm{VI}(\mathrm{GSVI}(\mathrm{G}) \cap \mathrm{Fix}(\mathrm{T}), \mathrm{B})}(\mathrm{p}-\lambda A \mathrm{p})$. Then, from (2.1), Proposition 2.2 (iii), $\beta$-inverse strong monotonicity of $A$, and $0<\lambda \leqslant 2 \beta$, it follows that

$$
\begin{aligned}
& \left\|\mathrm{P}_{\mathrm{VI}(\mathrm{GSVI}(\mathrm{G}) \cap \operatorname{Fix}(\mathrm{T}), \mathrm{B})}\left(z_{\mathrm{k}}-\lambda A z_{\mathrm{k}}\right)-\mathrm{p}\right\|^{2}
\end{aligned}
$$

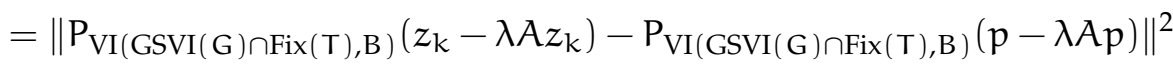

$$
\begin{aligned}
& \leqslant\left\|(\mathrm{I}-\lambda \mathrm{A}) z_{\mathrm{k}}-(\mathrm{I}-\lambda \mathrm{A}) \mathrm{p}\right\|^{2} \\
& \leqslant\left\|z_{k}-p\right\|^{2}+\lambda(\lambda-2 \beta)\left\|A z_{k}-A p\right\|^{2} \\
& \leqslant\left\|z_{\mathrm{k}}-\mathrm{p}\right\|^{2} \text {. }
\end{aligned}
$$

Utilizing (3.4), (3.8), (3.9) and the assumptions $0<\lambda \leqslant 2 \beta, \quad \sum_{k=0}^{\infty} \bar{\epsilon}_{k}<\infty$ we obtain that 


$$
\begin{aligned}
\left\|x_{k+1}-p\right\|= & \left\|\alpha_{k} u+\beta_{k} x_{k}+\gamma_{k} h_{k}-p\right\| \\
\leqslant & \alpha_{k}\|u-p\|+\beta_{k}\left\|x_{k}-p\right\|+\gamma_{k}\left\|h_{k}-p\right\| \\
\leqslant & \alpha_{k}\|u-p\|+\beta_{k}\left\|x_{k}-p\right\|+\gamma_{k}\left\|h_{k}-P_{V I(G S V I(G) \cap F i x(T), B)}\left(z_{k}-\lambda A z_{k}\right)\right\| \\
& +\gamma_{k}\left\|P_{V I(G S V I(G) \cap F i x(T), B)}\left(z_{k}-\lambda A z_{k}\right)-p\right\| \\
\leqslant & \alpha_{k}\|u-p\|+\beta_{k}\left\|x_{k}-p\right\|+\gamma_{k} \bar{\epsilon}_{k}+\gamma_{k}\left\|z_{k}-p\right\| \\
\leqslant & \alpha_{k}\|u-p\|+\beta_{k}\left\|x_{k}-p\right\|+\gamma_{k} \bar{\epsilon}_{k}+\gamma_{k}\left\|v_{k}-p\right\| \\
\leqslant & \alpha_{k}\|u-p\|+\beta_{k}\left\|x_{k}-p\right\|+\gamma_{k} \bar{\epsilon}_{k}+\gamma_{k} \max \left\{\left\|x_{k}-p\right\|, \frac{\|f(p)-p\|_{1}}{1-\rho}\right\} \\
\leqslant & \alpha_{k}\|u-p\|+\left(\beta_{k}+\gamma_{k}\right) \max \left\{\left\|x_{k}-p\right\|, \frac{\|f(p)-p\|}{1-\rho}\right\}+\gamma_{k} \bar{\epsilon}_{k} \\
= & \alpha_{k}\|u-p\|+\left(1-\alpha_{k}\right) \max \left\{\left\|x_{k}-p\right\|, \frac{\|f(p)-p\|}{1-\rho}\right\}+\gamma_{k} \bar{\epsilon}_{k} \\
\leqslant & \max \left\{\left\|x_{k}-p\right\|,\|u-p\|, \frac{\|f(p)-p\|}{1-\rho}\right\}+\bar{\epsilon}_{k} \\
\leqslant & \max \left\{\left\|x_{0}-p\right\|,\|u-p\|, \frac{\|f(p)-p\|}{1-\rho}\right\}+\sum_{j=0}^{k} \bar{\epsilon}_{j} \\
\leqslant & \max \left\{\left\|x_{0}-p\right\|,\|u-p\|, \frac{\|f(p)-p\|}{1-\rho}\right\}+\sum_{k=0}^{\infty} \bar{\epsilon}_{k} \\
< & \infty,
\end{aligned}
$$

which shows that the sequence $\left\{x_{k}\right\}$ is bounded, and so are the sequences $\left\{u_{k}\right\},\left\{\tilde{u}_{k}\right\},\left\{v_{k}\right\},\left\{y_{k}\right\}$, and $\left\{z_{k}\right\}$.

Lemma 3.6. Suppose that the hypotheses (H1)-(H4) hold. Assume that the sequences $\left\{v_{\mathrm{k}}\right\}$ and $\left\{z_{\mathrm{k}}\right\}$ are generated by Algorithm 3.2. Then, we have

$$
\left\|z_{k+1}-z_{k}\right\| \leqslant\left(1+\lambda_{k+1} \mathrm{~L}\right)\left\|v_{k+1}-v_{k}\right\|+\lambda_{k}\left\|B y_{k}\right\|+\lambda_{k+1}\left(\left\|B v_{k+1}\right\|+\left\|B y_{k+1}\right\|+\left\|B v_{k}\right\|\right) .
$$

Proof. Taking into account the L-Lipschitzian property of $B$, for each $x, y \in H$ we have

$$
\left\|\left(I-\lambda_{k} B\right) x-\left(I-\lambda_{k} B\right) y\right\|=\left\|x-y-\lambda_{k}(B x-B y)\right\| \leqslant\|x-y\|+\lambda_{k}\|B x-B y\| \leqslant\left(1+\lambda_{k} L\right)\|x-y\| .
$$

Combining this inequality with Proposition 2.2 (iii), we have

$$
\begin{aligned}
\left\|z_{k+1}-z_{k}\right\| & =\left\|P_{G S V I(G) \cap \operatorname{Fix}(\mathrm{T})}\left(v_{k+1}-\lambda_{k+1} B y_{k+1}\right)-P_{G S V I(G) \cap F i x(T)}\left(v_{k}-\lambda_{k} B y_{k}\right)\right\| \\
& \leqslant\left\|\left(v_{k+1}-\lambda_{k+1} B y_{k+1}\right)-v_{k}+\lambda_{k} B y_{k}\right\| \\
& =\left\|\left(v_{k+1}-\lambda_{k+1} B v_{k+1}\right)-\left(v_{k}-\lambda_{k+1} B v_{k}\right)+\lambda_{k+1}\left(B v_{k+1}-B y_{k+1}-B v_{k}\right)+\lambda_{k} B y_{k}\right\| \\
& \leqslant\left(1+\lambda_{k+1} \mathrm{~L}\right)\left\|v_{k+1}-v_{k}\right\|+\lambda_{k}\left\|B y_{k}\right\|+\lambda_{k+1}\left(\left\|B v_{k+1}\right\|+\left\|B y_{k+1}\right\|+\left\|B v_{k}\right\|\right) .
\end{aligned}
$$

This completes the proof.

Proposition 3.7. Let $\left\{x_{k}\right\}$ and $\left\{y_{k}\right\}$ be two bounded sequences in a real Banach space $X$. Let $\left\{\beta_{k}\right\}$ be a sequence in $[0,1]$. Suppose that $0<\liminf _{k \rightarrow \infty} \beta_{k} \leqslant \limsup _{k \rightarrow \infty} \beta_{k}<1, x_{k+1}=\left(1-\beta_{k}\right) y_{k}+\beta_{k} x_{k}$ and $\limsup _{k \rightarrow \infty}\left(\left\|y_{k+1}-y_{k}\right\|-\right.$ $\left.\left\|x_{k+1}-x_{k}\right\|\right) \leqslant 0$. Then, $\lim _{k \rightarrow \infty}\left\|y_{k}-x_{k}\right\|=0$.

Lemma 3.8. Suppose that the hypotheses $(\mathrm{H} 1)-(\mathrm{H} 4)$ hold. Assume that the sequence $\left\{x_{\mathrm{k}}\right\}$ is generated by Algorithm

3.2. Then, $\lim _{k \rightarrow \infty}\left\|x_{k+1}-x_{k}\right\|=0$. 
Proof. Since $\lim _{k \rightarrow \infty} \alpha_{k}=0, \lim _{k \rightarrow \infty} \beta_{k}=\xi \in\left(\zeta, \frac{1}{2}\right]$, and $\alpha_{k}+\beta_{k}+\gamma_{k}=1$, we get $\lim _{k \rightarrow \infty} \gamma_{k}=$ $\lim _{k \rightarrow \infty}\left(1-\alpha_{k}-\beta_{k}\right)=1-\xi \in[1 / 2,1-\zeta)$. Now, we write $x_{k+1}=\left(1-\beta_{k}\right) w_{k}+\beta_{k} x_{k}$ for all $k \geqslant 0$. Then, we have

$$
\begin{aligned}
w_{k+1}-w_{k} & =\frac{\alpha_{k+1} u+\gamma_{k+1} h_{k+1}}{1-\beta_{k+1}}-\frac{\alpha_{k} u+\gamma_{k} h_{k}}{1-\beta_{k}} \\
& =\left(\frac{\alpha_{k+1}}{1-\beta_{k+1}}-\frac{\alpha_{k}}{1-\beta_{k}}\right) u+\left(\frac{\gamma_{k+1}}{1-\beta_{k+1}}-\frac{\gamma_{k}}{1-\beta_{k}}\right) h_{k}+\frac{\gamma_{k+1}}{1-\beta_{k+1}}\left(h_{k+1}-h_{k}\right) .
\end{aligned}
$$

Note that, for $0<\lambda \leqslant 2 \beta$, we have from (2.1) that

$$
\begin{aligned}
& \| P_{\mathrm{VI}(\mathrm{GSVI}(\mathrm{G}) \cap \operatorname{Fix}(\mathrm{T}), \mathrm{B})}\left(z_{\mathrm{k}+1}-\lambda A z_{\mathrm{k}+1}\right)-\mathrm{P}_{\mathrm{VI}(\mathrm{GSVI}(\mathrm{G}) \cap \operatorname{Fix}(\mathrm{T}), \mathrm{B})\left(z_{\mathrm{k}}-\lambda A z_{\mathrm{k}}\right) \|^{2}} \\
& \quad \leqslant\left\|(\mathrm{I}-\lambda \mathrm{A}) z_{\mathrm{k}+1}-(\mathrm{I}-\lambda \mathrm{A}) z_{\mathrm{k}}\right\|^{2} \\
& \quad \leqslant\left\|z_{\mathrm{k}+1}-z_{\mathrm{k}}\right\|^{2}+\lambda(\lambda-2 \beta)\left\|A z_{\mathrm{k}+1}-A z_{\mathrm{k}}\right\|^{2} \\
& \quad \leqslant\left\|z_{\mathrm{k}+1}-z_{\mathrm{k}}\right\|^{2}
\end{aligned}
$$

Then, utilizing (3.10) and (3.11) we get

$$
\begin{aligned}
\| & w_{k+1}-w_{k} \| \\
\leqslant & \left|\frac{\alpha_{k+1}}{1-\beta_{k+1}}-\frac{\alpha_{k}}{1-\beta_{k}}\right|\|u\|+\left|\frac{\gamma_{k+1}}{1-\beta_{k+1}}-\frac{\gamma_{k}}{1-\beta_{k}}\right|\left(\left\|P_{V I(G S V I(G) \cap F i x(T), B)}\left(z_{k}-\lambda A z_{k}\right)\right\|+\bar{\epsilon}_{k}\right) \\
& +\frac{\gamma_{k+1}}{1-\beta_{k+1}}\left\|z_{k+1}-z_{k}\right\|+\frac{\gamma_{k+1}}{1-\beta_{k+1}}\left(\bar{\epsilon}_{k+1}+\bar{\epsilon}_{k}\right) \\
\leqslant & \left|\frac{\alpha_{k+1}}{1-\beta_{k+1}}-\frac{\alpha_{k}}{1-\beta_{k}}\right|\|u\|+\left|\frac{\gamma_{k+1}}{1-\beta_{k+1}}-\frac{\gamma_{k}}{1-\beta_{k}}\right|\left(\left\|P_{V I(G S V I(G) \cap F i x(T), B)}\left(z_{k}-\lambda A z_{k}\right)\right\|+\bar{\epsilon}_{k}\right) \\
& +\frac{\gamma_{k+1}\left(1+\lambda_{k+1} L\right)}{1-\beta_{k+1}}\left\|v_{k+1}-v_{k}\right\|+\frac{\gamma_{k+1}}{1-\beta_{k+1}}\left(\bar{\epsilon}_{k+1}+\bar{\epsilon}_{k}\right) \\
& +\frac{\gamma_{k+1}}{1-\beta_{k+1}}\left(\lambda_{k+1}\left(\left\|B v_{k+1}\right\|+\left\|B y_{k+1}\right\|+\left\|B v_{k}\right\|\right)+\lambda_{k}\left\|B y_{k}\right\|\right) \\
= & \left|\frac{\alpha_{k+1}}{1-\beta_{k+1}}-\frac{\alpha_{k}}{1-\beta_{k}}\right|\|u\|+\left|\frac{\alpha_{k+1}}{1-\beta_{k+1}}-\frac{\alpha_{k}}{1-\beta_{k}}\right|\left(\left\|P_{V I(G S V I(G) \cap F i x(T), B)}\left(z_{k}-\lambda A z_{k}\right)\right\|+\bar{\epsilon}_{k}\right) \\
& +\frac{\gamma_{k+1}\left(1+\lambda_{k+1} L\right)}{\alpha_{k+1}+\gamma_{k+1}}\left\|v_{k+1}-v_{k}\right\|+\frac{\gamma_{k+1}}{\alpha_{k+1}+\gamma_{k+1}}\left(\bar{\epsilon}_{k+1}+\bar{\epsilon}_{k}\right) \\
& +\frac{\gamma_{k+1}}{\alpha_{k+1}+\gamma_{k+1}}\left(\lambda_{k+1}\left(\left\|B v_{k+1}\right\|+\left\|B y_{k+1}\right\|+\left\|B v_{k}\right\|\right)+\lambda_{k}\left\|B y_{k}\right\|\right) \\
\leqslant & \left(\frac{\left|\alpha_{k+1}-\alpha_{k}\right|}{1-\beta_{k+1}}+\frac{\alpha_{k}\left|\beta_{k+1}-\beta_{k}\right|}{\left(1-\beta_{k+1}\right)\left(1-\beta_{k}\right)}\right)\left(\|u\|+\left\|P_{V I(G S V I(G) \cap F i x(T), B)}\left(z_{k}-\lambda A z_{k}\right)\right\|+\bar{\epsilon}_{k}\right) \\
& +\left\|v_{k+1}-v_{k}\right\|+\lambda_{k+1} L\left\|v_{k+1}-v_{k}\right\|+\bar{\epsilon}_{k+1}+\bar{\epsilon}_{k} \\
& +\lambda_{k+1}\left(\left\|B v_{k+1}\right\|+\left\|B y_{k+1}\right\|+\left\|B v_{k}\right\|\right)+\lambda_{k}\left\|B y_{k}\right\| \\
\leqslant & \left\|v_{k+1}-v_{k}\right\|+\left(\left|\alpha_{k+1}-\alpha_{k}\right|+\left|\beta_{k+1}-\beta_{k}\right|\right) \frac{\|u\|+\left\|P_{V I(G S V I(G) \cap F i x(T), B)}\left(z_{k}-\lambda A z_{k}\right)\right\|+\bar{\epsilon}_{k}}{1-b} \\
& +\bar{\epsilon}_{k+1}+\bar{\epsilon}_{k}+\lambda_{k+1}\left(L\left\|v_{k+1}-v_{k}\right\|+\left\|B v_{k+1}\right\|+\left\|B y_{k+1}\right\|+\left\|B v_{k}\right\|\right)+\lambda_{k}\left\|B y_{k}\right\| .
\end{aligned}
$$

For simplicity, we write $S=\mu \mathrm{I}+(1-\mu) \mathrm{T}$ for $0 \leqslant \zeta \leqslant \mu<1$. According to Lemma 2.9 we know that $S$ is a nonexpansive mapping. It is clear that $\operatorname{Fix}(S)=\operatorname{Fix}(T)$. Also, we write $v_{k}=\beta_{k} x_{k}+\left(1-\beta_{k}\right) \tilde{w}_{k}$ for all $k \geqslant 0$, where

$$
\tilde{w}_{k}=\frac{v_{k}-\beta_{k} x_{k}}{1-\beta_{k}}=\frac{\alpha_{k} u_{k}+\gamma_{k}\left[\mu G x_{k}+(1-\mu) T G x_{k}\right]}{1-\beta_{k}}=\frac{\alpha_{k} u_{k}+\gamma_{k} S G x_{k}}{1-\beta_{k}}
$$


Observe that

$$
\begin{aligned}
& \left\|\tilde{w}_{k+1}-\tilde{w}_{k}\right\| \\
& \quad=\left\|\frac{\alpha_{k+1} u_{k+1}+\gamma_{k+1} S G x_{k+1}}{1-\beta_{k+1}}-\frac{\alpha_{k} u_{k}+\gamma_{k} S G x_{k}}{1-\beta_{k}}\right\| \\
& \quad \leqslant\left\|\frac{\alpha_{k+1}}{1-\beta_{k+1}} u_{k+1}-\frac{\alpha_{k}}{1-\beta_{k}} u_{k}\right\|+\left|\frac{\gamma_{k+1}}{1-\beta_{k+1}}-\frac{\gamma_{k}}{1-\beta_{k}}\right|\left\|S G x_{k+1}\right\|+\frac{\gamma_{k}}{1-\beta_{k}}\left\|S G x_{k+1}-S G x_{k}\right\| \\
& \quad \leqslant \frac{\alpha_{k+1}}{1-\beta_{k+1}}\left\|u_{k+1}\right\|+\frac{\alpha_{k}}{1-\beta_{k}}\left\|u_{k}\right\|+\left|\frac{\gamma_{k+1}}{1-\beta_{k+1}}-\frac{\gamma_{k}}{1-\beta_{k}}\right|\left\|S G x_{k+1}\right\|+\frac{\gamma_{k}}{1-\beta_{k}}\left\|x_{k+1}-x_{k}\right\| .
\end{aligned}
$$

Moreover, simple calculations show that

$$
\begin{aligned}
v_{k+1}-v_{k} & =\beta_{k+1} x_{k+1}+\left(1-\beta_{k+1}\right) \tilde{w}_{k+1}-\beta_{k} x_{k}-\left(1-\beta_{k}\right) \tilde{w}_{k} \\
& =\left(\beta_{k+1}-\beta_{k}\right)\left(x_{k+1}-\tilde{w}_{k+1}\right)+\beta_{k}\left(x_{k+1}-x_{k}\right)+\left(1-\beta_{k}\right)\left(\tilde{w}_{k+1}-\tilde{w}_{k}\right),
\end{aligned}
$$

which together with (3.13), leads to

$$
\begin{aligned}
\left\|v_{k+1}-v_{k}\right\| \leqslant & \left|\beta_{k+1}-\beta_{k}\right| \mid x_{k+1}-\tilde{w}_{k+1}\left\|+\beta_{k}\right\| x_{k+1}-x_{k}\left\|+\left(1-\beta_{k}\right)\right\| \tilde{w}_{k+1}-\tilde{w}_{k} \| \\
\leqslant & \left|\beta_{k+1}-\beta_{k}\right| \mid x_{k+1}-\tilde{w}_{k+1}\left\|+\beta_{k}\right\| x_{k+1}-x_{k} \|+\left(1-\beta_{k}\right)\left[\frac{\alpha_{k+1}}{1-\beta_{k+1}}\left\|u_{k+1}\right\|\right. \\
& \left.+\frac{\alpha_{k}}{1-\beta_{k}}\left\|u_{k}\right\|+\left|\frac{\gamma_{k+1}}{1-\beta_{k+1}}-\frac{\gamma_{k}}{1-\beta_{k}}\right|\left\|S G x_{k+1}\right\|+\frac{\gamma_{k}}{1-\beta_{k}}\left\|x_{k+1}-x_{k}\right\|\right] \\
= & \left|\beta_{k+1}-\beta_{k}\right|\left\|x_{k+1}-\tilde{w}_{k+1}\right\|+\left(\beta_{k}+\gamma_{k}\right)\left\|x_{k+1}-x_{k}\right\| \\
& +\left(1-\beta_{k}\right)\left[\frac{\alpha_{k+1}}{1-\beta_{k+1}}\left\|u_{k+1}\right\|+\frac{\alpha_{k}}{1-\beta_{k}}\left\|u_{k}\right\|+\left|\frac{\gamma_{k+1}}{1-\beta_{k+1}}-\frac{\gamma_{k}}{1-\beta_{k}}\right|\left\|S G x_{k+1}\right\|\right] \\
\leqslant & \left|\beta_{k+1}-\beta_{k}\right| \mid x_{k+1}-\tilde{w}_{k+1}\|+\| x_{k+1}-x_{k}\left\|+\frac{\alpha_{k+1}}{1-\beta_{k+1}}\right\| u_{k+1}\left\|+\frac{\alpha_{k}}{1-\beta_{k}}\right\| u_{k} \| \\
& +\left|\frac{\gamma_{k+1}}{1-\beta_{k+1}}-\frac{\gamma_{k}}{1-\beta_{k}}\right|\left\|S G x_{k+1}\right\| .
\end{aligned}
$$

Combining (3.12) and (3.14), we obtain

$$
\begin{aligned}
& \left\|w_{k+1}-w_{k}\right\| \leqslant\left\|v_{k+1}-v_{k}\right\|+\left(\left|\alpha_{k+1}-\alpha_{k}\right|+\left|\beta_{k+1}-\beta_{k}\right|\right) \frac{\|u\|+\left\|P_{V I(G S V I(G) \cap F i x(T), B)}\left(z_{k}-\lambda A z_{k}\right)\right\|+\bar{\epsilon}_{k}}{1-b} \\
& +\bar{\epsilon}_{k+1}+\bar{\epsilon}_{k}+\lambda_{k+1}\left(\mathrm{~L}\left\|v_{k+1}-v_{k}\right\|+\left\|B v_{k+1}\right\|+\left\|B y_{k+1}\right\|+\left\|B v_{k}\right\|\right)+\lambda_{k}\left\|B y_{k}\right\| \\
& \leqslant\left|\beta_{k+1}-\beta_{k}\right|\left\|x_{k+1}-\tilde{w}_{k+1}\right\|+\left\|x_{k+1}-x_{k}\right\|+\frac{\alpha_{k+1}}{1-\beta_{k+1}}\left\|u_{k+1}\right\|+\frac{\alpha_{k}}{1-\beta_{k}}\left\|u_{k}\right\| \\
& +\left|\frac{\gamma_{k+1}}{1-\beta_{k+1}}-\frac{\gamma_{k}}{1-\beta_{k}}\right|\left|S G x_{k+1}\right| \mid+\left(\left|\alpha_{k+1}-\alpha_{k}\right|+\left|\beta_{k+1}-\beta_{k}\right|\right) \\
& \times \frac{\|u\|+\left\|P_{\mathrm{VI}(\mathrm{GSVI}(\mathrm{G}) \cap \operatorname{Fix}(\mathrm{T}), \mathrm{B})}\left(z_{\mathrm{k}}-\lambda \mathrm{A} z_{\mathrm{k}}\right)\right\|+\bar{\epsilon}_{\mathrm{k}}}{1-\mathrm{b}}+\bar{\epsilon}_{\mathrm{k}+1}+\bar{\epsilon}_{\mathrm{k}} \\
& +\lambda_{k+1}\left(\mathrm{~L}\left\|v_{k+1}-v_{k}\right\|+\left\|\mathrm{B} v_{k+1}\right\|+\left\|B y_{k+1}\right\|+\left\|\mathrm{B} v_{k}\right\|\right)+\lambda_{k}\left\|B y_{k}\right\|,
\end{aligned}
$$

which immediately yields

$$
\begin{aligned}
& \left\|w_{k+1}-w_{k}\right\|-\left\|x_{k+1}-x_{k}\right\| \leqslant\left|\beta_{k+1}-\beta_{k}\right|\left\|x_{k+1}-\tilde{w}_{k+1}\right\|+\frac{\alpha_{k+1}}{1-\beta_{k+1}}\left\|u_{k+1}\right\|+\frac{\alpha_{k}}{1-\beta_{k}}\left\|u_{k}\right\| \\
& +\left|\frac{\gamma_{k+1}}{1-\beta_{k+1}}-\frac{\gamma_{k}}{1-\beta_{k}}\right|\left|S G x_{k+1}\right| \mid+\left(\left|\alpha_{k+1}-\alpha_{k}\right|+\left|\beta_{k+1}-\beta_{k}\right|\right)
\end{aligned}
$$

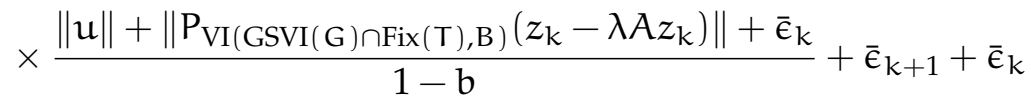

$$
\begin{aligned}
& +\lambda_{k+1}\left(\mathrm{~L}\left\|v_{\mathrm{k}+1}-v_{\mathrm{k}}\right\|+\left\|\mathrm{B} v_{\mathrm{k}+1}\right\|+\left\|\mathrm{B} \mathrm{y}_{\mathrm{k}+1}\right\|+\left\|\mathrm{B} v_{\mathrm{k}}\right\|\right)+\lambda_{\mathrm{k}}\left\|\mathrm{B} \mathrm{y}_{\mathrm{k}}\right\| \text {. }
\end{aligned}
$$


Since $\lim _{k \rightarrow \infty} \alpha_{k}=0, \lim _{k \rightarrow \infty} \beta_{k}=\xi \in\left(\zeta, \frac{1}{2}\right], \alpha_{k}+\beta_{k}+\gamma_{k}=1, \lim _{k \rightarrow \infty} \bar{\epsilon}_{k}=0$, and $\lim _{k \rightarrow \infty} \lambda_{k}=0$, we conclude from the boundedness of the sequences $\left\{u_{k}\right\},\left\{v_{k}\right\},\left\{x_{k}\right\},\left\{y_{k}\right\},\left\{z_{k}\right\}$, and $\left\{\tilde{w}_{k}\right\}$ that

$$
\limsup _{k \rightarrow \infty}\left(\left\|w_{k+1}-w_{k}\right\|-\left\|x_{k+1}-x_{k}\right\|\right) \leqslant 0
$$

Therefore, by Proposition 3.1 we have

$$
\lim _{k \rightarrow \infty}\left\|w_{k}-x_{k}\right\|=0,
$$

which together with $x_{k+1}=\beta_{k} x_{k}+\left(1-\beta_{k}\right) w_{k}$, implies that

$$
\lim _{k \rightarrow \infty}\left\|x_{k+1}-x_{k}\right\|=\lim _{k \rightarrow \infty}\left(1-\beta_{k}\right)\left\|w_{k}-x_{k}\right\|=0 .
$$

Lemma 3.9. Suppose that the hypotheses (H1)-(H4) hold. Then for any $\mathrm{p} \in \Omega:=\operatorname{VI}(\operatorname{VI}(\mathrm{GSVI}(\mathrm{G}) \cap \operatorname{Fix}(\mathrm{T}), \mathrm{B}), \mathrm{A})$ we have

$$
\begin{aligned}
\left\|x_{k+1}-p\right\|^{2} \leqslant & \alpha_{k}\|u-p\|^{2}+\beta_{k}\left\|x_{k}-p\right\|^{2}+\gamma_{k}\left\|v_{k}-p\right\|^{2}+2 \gamma_{k} \bar{\epsilon}_{k}\left\|z_{k}-p\right\| \\
& +\gamma_{k} \bar{\epsilon}_{k}^{2}-\gamma_{k}\left(1-\lambda_{k} L\right)\left(\left\|v_{k}-y_{k}\right\|^{2}+\left\|y_{k}-z_{k}\right\|^{2}\right) .
\end{aligned}
$$

Moreover, we have

$$
\lim _{k \rightarrow \infty}\left\|P_{\operatorname{VI}(G S V I(G) \cap F i x(T), B)}\left(z_{k}-\lambda_{k} A z_{k}\right)-z_{k}\right\|=0
$$

and

$$
\lim _{k \rightarrow \infty}\left\|P_{V I(G S V I(G) \cap \operatorname{Fix}(T), B)}\left(y_{k}-\lambda_{k} A y_{k}\right)-y_{k}\right\|=0 .
$$

Proof. By Lemma 3.3, we know that

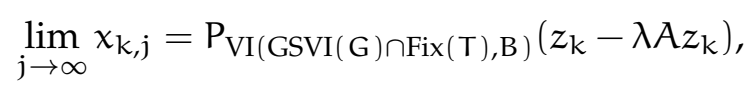

which together with $0<\lambda \leqslant 2 \beta$, inequality (3.2), $\lim _{k \rightarrow \infty} \beta_{k}=\xi \in\left(\zeta, \frac{1}{2}\right]$, and

$$
p \in \Omega:=\operatorname{VI}(\operatorname{VI}(\operatorname{GSVI}(G) \cap \operatorname{Fix}(T), B), A),
$$

implies that

$$
\begin{aligned}
\left\|x_{k+1}-p\right\|^{2}= & \left\|\alpha_{k} u+\beta_{k} x_{k}+\gamma_{k} h_{k}-p\right\|^{2} \\
\leqslant & \alpha_{k}\|u-p\|^{2}+\beta_{k}\left\|x_{k}-p\right\|^{2}+\gamma_{k}\left\|h_{k}-p\right\|^{2} \\
\leqslant & \alpha_{k}\|u-p\|^{2}+\beta_{k}\left\|x_{k}-p\right\|^{2}+\gamma_{k}\left(\left\|P_{V I(G S V I(G) \cap F i x(T), B)}\left(z_{k}-\lambda A z_{k}\right)-p\right\|+\bar{\epsilon}_{k}\right)^{2} \\
= & \gamma_{k}\left(\| P_{V I}(G S V I(G) \cap \operatorname{Fix}(T), B)\left(z_{k}-\lambda A z_{k}\right)-P_{V I(G S V I(G) \cap F i x(T), B)}\right. \\
& \left.+\alpha_{k}\|u-p\|^{2}+\beta_{k}\left\|x_{k}-p\right\|^{2}(p-\lambda A p) \|+\bar{\epsilon}_{k}\right)^{2} \\
\leqslant & \alpha_{k}\|u-p\|^{2}+\beta_{k}\left\|x_{k}-p\right\|^{2}+\gamma_{k}\left(\left\|(I-\lambda A) z_{k}-(I-\lambda A) p\right\|+\bar{\epsilon}_{k}\right)^{2} \\
\leqslant & \alpha_{k}\|u-p\|^{2}+\beta_{k}\left\|x_{k}-p\right\|^{2}+\gamma_{k}\left(\left\|z_{k}-p\right\|+\bar{\epsilon}_{k}\right)^{2} \\
= & \alpha_{k}\|u-p\|^{2}+\beta_{k}\left\|x_{k}-p\right\|^{2}+\gamma_{k}\left\|z_{k}-p\right\|^{2}+2 \gamma_{k} \bar{\epsilon}_{k}\left\|z_{k}-p\right\|+\gamma_{k} \bar{\epsilon}_{k}^{2} \\
\leqslant & \alpha_{k}\|u-p\|^{2}+\beta_{k}\left\|x_{k}-p\right\|^{2}+2 \gamma_{k} \bar{\epsilon}_{k}\left\|z_{k}-p\right\|+\gamma_{k} \bar{\epsilon}_{k}^{2} \\
& +\gamma_{k}\left(\left\|v_{k}-p\right\|^{2}-\left(1-\lambda_{k} L\right)\left\|v_{k}-y_{k}\right\|^{2}-\left(1-\lambda_{k} L\right)\left\|y_{k}-z_{k}\right\|^{2}\right) \\
= & \alpha_{k}\|u-p\|^{2}+\beta_{k}\left\|x_{k}-p\right\|^{2}+\gamma_{k}\left\|v_{k}-p\right\|^{2}+2 \gamma_{k} \bar{\epsilon}_{k}\left\|z_{k}-p\right\|+\gamma_{k} \bar{\epsilon}_{k}^{2} \\
& -\gamma_{k}\left(1-\lambda_{k} L\right)\left(\left\|v_{k}-y_{k}\right\|^{2}+\left\|y_{k}-z_{k}\right\|^{2}\right) .
\end{aligned}
$$

Next we claim that $\left\|x_{k}-v_{k}\right\| \rightarrow 0$ as $n \rightarrow \infty$. Indeed, for simplicity, we write $q_{k}=G x_{k}, \tilde{x}_{k}=P_{C}(I-$ $\left.v_{2} F_{2}\right) x_{k}$ and $\tilde{p}=P_{C}\left(I-v_{2} F_{2}\right) p$. Then $q_{k}=P_{C}\left(I-v_{1} F_{1}\right) \tilde{x}_{k}$. Utilizing Algorithm 3.2, we obtain from (3.5) 
and (3.6) that

$$
\begin{aligned}
\left\|v_{k}-p\right\|^{2}= & \left\|\alpha_{k}\left(u_{k}-p\right)+\beta_{k}\left(x_{k}-p\right)+\gamma_{k}\left(\tilde{u}_{k}-p\right)\right\|^{2} \\
\leqslant & \alpha_{k}\left\|u_{k}-p\right\|^{2}+\beta_{k}\left\|x_{k}-p\right\|^{2}+\gamma_{k}\left\|\tilde{u}_{k}-p\right\|^{2} \\
\leqslant & \alpha_{k}\left\|u_{k}-p\right\|^{2}+\beta_{k}\left\|x_{k}-p\right\|^{2}+\gamma_{k}\left[\left\|G x_{k}-p\right\|^{2}-(1-\mu)(\mu-\zeta)\left\|G x_{k}-T G x_{k}\right\|^{2}\right] \\
\leqslant & \alpha_{k}\left\|u_{k}-p\right\|^{2}+\beta_{k}\left\|x_{k}-p\right\|^{2}+\gamma_{k}\left[\left\|x_{k}-p\right\|^{2}+v_{2}\left(v_{2}-2 \zeta_{2}\right)\left\|F_{2} x_{k}-F_{2} p\right\|^{2}\right. \\
& \left.+v_{1}\left(v_{1}-2 \zeta_{1}\right)\left\|F_{1} \tilde{x}_{k}-F_{1} \tilde{p}\right\|^{2}-(1-\mu)(\mu-\zeta)\left\|G x_{k}-T G x_{k}\right\|^{2}\right] \\
= & \alpha_{k}\left\|u_{k}-p\right\|^{2}+\left(\beta_{k}+\gamma_{k}\right)\left\|x_{k}-p\right\|^{2}+\gamma_{k}\left[v_{2}\left(v_{2}-2 \zeta_{2}\right)\left\|F_{2} x_{k}-F_{2} p\right\|^{2}\right. \\
& \left.+v_{1}\left(v_{1}-2 \zeta_{1}\right)\left\|F_{1} \tilde{x}_{k}-F_{1} \tilde{p}\right\|^{2}-(1-\mu)(\mu-\zeta)\left\|G x_{k}-T G x_{k}\right\|^{2}\right] \\
\leqslant & \alpha_{k}\left\|u_{k}-p\right\|^{2}+\left\|x_{k}-p\right\|^{2}-\gamma_{k}\left[v_{2}\left(2 \zeta_{2}-v_{2}\right)\left\|F_{2} x_{k}-F_{2} p\right\|^{2}\right. \\
& \left.+v_{1}\left(2 \zeta_{1}-v_{1}\right)\left\|F_{1} \tilde{x}_{k}-F_{1} \tilde{p}\right\|^{2}+(1-\mu)(\mu-\zeta)\left\|G x_{k}-T G x_{k}\right\|^{2}\right] .
\end{aligned}
$$

Combining (3.15) and (3.16) we get

$$
\begin{aligned}
\left\|x_{k+1}-p\right\|^{2} \leqslant & \alpha_{k}\|u-p\|^{2}+\beta_{k}\left\|x_{k}-p\right\|^{2}+\gamma_{k}\left\|v_{k}-p\right\|^{2}+2 \gamma_{k} \bar{\epsilon}_{k}\left\|z_{k}-p\right\|+\gamma_{k} \bar{\epsilon}_{k}^{2} \\
& -\gamma_{k}\left(1-\lambda_{k} L\right)\left(\left\|v_{k}-y_{k}\right\|^{2}+\left\|y_{k}-z_{k}\right\|^{2}\right) \\
\leqslant & \alpha_{k}\|u-p\|^{2}+\beta_{k}\left\|x_{k}-p\right\|^{2}+\gamma_{k}\left\{\alpha_{k}\left\|u_{k}-p\right\|^{2}+\left\|x_{k}-p\right\|^{2}\right. \\
& -\gamma_{k}\left[v_{2}\left(2 \zeta_{2}-v_{2}\right)\left\|F_{2} x_{k}-F_{2} p\right\|^{2}+v_{1}\left(2 \zeta_{1}-v_{1}\right)\left\|F_{1} \tilde{x}_{k}-F_{1} \tilde{p}\right\|^{2}\right. \\
& \left.\left.+(1-\mu)(\mu-\zeta)\left\|G x_{k}-T G x_{k}\right\|^{2}\right]\right\}+2 \gamma_{k} \bar{\epsilon}_{k}\left\|z_{k}-p\right\| \\
& +\gamma_{k} \bar{\epsilon}_{k}^{2}-\gamma_{k}\left(1-\lambda_{k} L\right)\left(\left\|v_{k}-y_{k}\right\|^{2}+\left\|y_{k}-z_{k}\right\|^{2}\right) \\
\leqslant & \alpha_{k}\|u-p\|^{2}+\alpha_{k}\left\|u_{k}-p\right\|^{2}+\left(\beta_{k}+\gamma_{k}\right)\left\|x_{k}-p\right\|^{2} \\
& -\gamma_{k}^{2}\left[v_{2}\left(2 \zeta_{2}-v_{2}\right)\left\|F_{2} x_{k}-F_{2} p\right\|^{2}+v_{1}\left(2 \zeta_{1}-v_{1}\right)\left\|F_{1} \tilde{x}_{k}-F_{1} \tilde{p}\right\|^{2}\right. \\
& \left.+(1-\mu)(\mu-\zeta)\left\|G x_{k}-T G x_{k}\right\|^{2}\right]+2 \bar{\epsilon}_{k}\left\|z_{k}-p\right\| \\
& +\bar{\epsilon}_{k}^{2}-\gamma_{k}\left(1-\lambda_{k} L\right)\left(\left\|v_{k}-y_{k}\right\|^{2}+\left\|y_{k}-z_{k}\right\|^{2}\right) \\
\leqslant & \alpha_{k}\|u-p\|^{2}+\alpha_{k}\left\|u_{k}-p\right\|^{2}+\left\|x_{k}-p\right\|^{2}-\gamma_{k}^{2}\left[v_{2}\left(2 \zeta_{2}-v_{2}\right)\left\|F_{2} x_{k}-F_{2} p\right\|^{2}\right. \\
& \left.+v_{1}\left(2 \zeta_{1}-v_{1}\right)\left\|F_{1} \tilde{x}_{k}-F_{1} \tilde{p}\right\|^{2}+(1-\mu)(\mu-\zeta)\left\|G x_{k}-T G x_{k}\right\|^{2}\right] \\
& +2 \bar{\epsilon}_{k}\left\|z_{k}-p\right\|+\bar{\epsilon}_{k}^{2}-\gamma_{k}\left(1-\lambda_{k} L\right)\left(\left\|v_{k}-y_{k}\right\|^{2}+\left\|y_{k}-z_{k}\right\|^{2}\right),
\end{aligned}
$$

which immediately yields

$$
\begin{aligned}
\gamma_{k}(1 & \left.-\lambda_{k} L\right)\left(\left\|v_{k}-y_{k}\right\|^{2}+\left\|y_{k}-z_{k}\right\|^{2}\right)+\gamma_{k}^{2}\left[v_{2}\left(2 \zeta_{2}-v_{2}\right)\left\|F_{2} x_{k}-F_{2} p\right\|^{2}\right. \\
& \left.+v_{1}\left(2 \zeta_{1}-v_{1}\right)\left\|F_{1} \tilde{x}_{k}-F_{1} \tilde{p}\right\|^{2}+(1-\mu)(\mu-\zeta)\left\|G x_{k}-T G x_{k}\right\|^{2}\right] \\
\leqslant & \alpha_{k}\|u-p\|^{2}+\alpha_{k}\left\|u_{k}-p\right\|^{2}+\left\|x_{k}-p\right\|^{2}-\left\|x_{k+1}-p\right\|^{2}+2 \bar{\epsilon}_{k}\left\|z_{k}-p\right\|+\bar{\epsilon}_{k}^{2} \\
\leqslant & \alpha_{k}\left(\|u-p\|^{2}+\left\|u_{k}-p\right\|^{2}\right)+\left\|x_{k}-x_{k+1}\right\|\left(\left\|x_{k}-p\right\|+\left\|x_{k+1}-p\right\|\right)+2 \bar{\epsilon}_{k}\left\|z_{k}-p\right\|+\bar{\epsilon}_{k}^{2} .
\end{aligned}
$$

Since $\alpha_{k}+\beta_{k}+\gamma_{k}=1, \alpha_{k} \rightarrow 0, \beta_{k} \rightarrow \xi \in\left(\zeta, \frac{1}{2}\right], \bar{\epsilon}_{k} \rightarrow 0, \lambda_{k} \rightarrow 0$, and $\left\|x_{k+1}-x_{k}\right\| \rightarrow 0$ (due to Lemma 3.8), we deduce from the boundedness of $\left\{x_{k}\right\},\left\{u_{k}\right\}$ and $\left\{z_{k}\right\}$ that

$$
\left\{\begin{array}{l}
\lim _{k \rightarrow \infty}\left\|F_{2} x_{k}-F_{2} p\right\|=\lim _{k \rightarrow \infty}\left\|F_{1} \tilde{x}_{k}-F_{1} \tilde{p}\right\|=0, \\
\lim _{k \rightarrow \infty}\left\|G x_{k}-T G x_{k}\right\|=\lim _{k \rightarrow \infty}\left\|v_{k}-y_{k}\right\|=\lim _{k \rightarrow \infty}\left\|y_{k}-z_{k}\right\|=0 .
\end{array}\right.
$$

On the other hand, in terms of the firm nonexpansivity of $P_{C}$ and the $\zeta_{i}$-inverse strong monotonicity of $F_{i}$ for $i=1,2$, we obtain from $v_{i} \in\left(0,2 \zeta_{i}\right), i=1,2$ and (3.6) that

$$
\left\|\tilde{x}_{k}-\tilde{p}\right\|^{2}=\left\|P_{C}\left(I-v_{2} F_{2}\right) x_{k}-P_{C}\left(I-v_{2} F_{2}\right) p\right\|^{2}
$$




$$
\begin{aligned}
\leqslant & \left\langle\left(I-v_{2} F_{2}\right) x_{k}-\left(I-v_{2} F_{2}\right) p, \tilde{x}_{k}-\tilde{p}\right\rangle \\
= & \frac{1}{2}\left[\left\|\left(I-v_{2} F_{2}\right) x_{k}-\left(I-v_{2} F_{2}\right) p\right\|^{2}+\left\|\tilde{x}_{k}-\tilde{p}\right\|^{2}-\left\|\left(I-v_{2} F_{2}\right) x_{k}-\left(I-v_{2} F_{2}\right) p-\left(\tilde{x}_{k}-\tilde{p}\right)\right\|^{2}\right] \\
\leqslant & \frac{1}{2}\left[\left\|x_{k}-p\right\|^{2}+\left\|\tilde{x}_{k}-\tilde{p}\right\|^{2}-\left\|\left(x_{k}-\tilde{x}_{k}\right)-v_{2}\left(F_{2} x_{k}-F_{2} p\right)-(p-\tilde{p})\right\|^{2}\right] \\
= & \frac{1}{2}\left[\left\|x_{k}-p\right\|^{2}+\left\|\tilde{x}_{k}-\tilde{p}\right\|^{2}-\left\|\left(x_{k}-\tilde{x}_{k}\right)-(p-\tilde{p})\right\|^{2}\right. \\
& \left.+2 v_{2}\left\langle\left(x_{k}-\tilde{x}_{k}\right)-(p-\tilde{p}), F_{2} x_{k}-F_{2} p\right\rangle-v_{2}^{2}\left\|F_{2} x_{k}-F_{2} p\right\|^{2}\right],
\end{aligned}
$$

and

$$
\begin{aligned}
\left\|q_{k}-p\right\|^{2}= & \left\|P_{C}\left(I-v_{1} F_{1}\right) \tilde{x}_{k}-P_{C}\left(I-v_{1} F_{1}\right) \tilde{p}\right\|^{2} \\
\leqslant & \left\langle\left(I-v_{1} F_{1}\right) \tilde{x}_{k}-\left(I-v_{1} F_{1}\right) \tilde{p}, q_{k}-p\right\rangle \\
= & \frac{1}{2}\left[\left\|\left(I-v_{1} F_{1}\right) \tilde{x}_{k}-\left(I-v_{1} F_{1}\right) \tilde{p}\right\|^{2}+\left\|q_{k}-p\right\|^{2}-\left\|\left(I-v_{1} F_{1}\right) \tilde{x}_{k}-\left(I-v_{1} F_{1}\right) \tilde{p}-\left(q_{k}-p\right)\right\|^{2}\right] \\
\leqslant & \frac{1}{2}\left[\left\|\tilde{x}_{k}-\tilde{p}\right\|^{2}+\left\|q_{k}-p\right\|^{2}-\left\|\left(\tilde{x}_{k}-q_{k}\right)+(p-\tilde{p})\right\|^{2}+2 v_{1}\left\langle F_{1} \tilde{x}_{k}-F_{1} \tilde{p},\left(\tilde{x}_{k}-q_{k}\right)+(p-\tilde{p})\right\rangle\right. \\
& \left.-v_{1}^{2}\left\|F_{1} \tilde{x}_{k}-F_{1} \tilde{p}\right\|^{2}\right] \\
\leqslant & \frac{1}{2}\left[\left\|x_{k}-p\right\|^{2}+\left\|q_{k}-p\right\|^{2}-\left\|\left(\tilde{x}_{k}-q_{k}\right)+(p-\tilde{p})\right\|^{2}+2 v_{1}\left\langle F_{1} \tilde{x}_{k}-F_{1} \tilde{p},\left(\tilde{x}_{k}-q_{k}\right)+(p-\tilde{p})\right\rangle\right] .
\end{aligned}
$$

Thus, we have

$$
\begin{aligned}
\left\|\tilde{x}_{k}-\tilde{p}\right\|^{2} \leqslant & \left\|x_{k}-p\right\|^{2}-\left\|\left(x_{k}-\tilde{x}_{k}\right)-(p-\tilde{p})\right\|^{2}+2 v_{2}\left\langle\left(x_{k}-\tilde{x}_{k}\right)-(p-\tilde{p}), F_{2} x_{k}-F_{2} p\right\rangle \\
& -v_{2}^{2}\left\|F_{2} x_{k}-F_{2} p\right\|^{2}
\end{aligned}
$$

and

$$
\left\|q_{k}-p\right\|^{2} \leqslant\left\|x_{k}-p\right\|^{2}-\left\|\left(\tilde{x}_{k}-q_{k}\right)+(p-\tilde{p})\right\|^{2}+2 v_{1}\left\|F_{1} \tilde{x}_{k}-F_{1} \tilde{p}\right\|\left\|\left(\tilde{x}_{k}-q_{k}\right)+(p-\tilde{p})\right\| .
$$

In the meantime, utilizing (3.16) and (3.18) we obtain

$$
\begin{aligned}
\left\|v_{k}-p\right\|^{2} \leqslant & \alpha_{k}\left\|u_{k}-p\right\|^{2}+\beta_{k}\left\|x_{k}-p\right\|^{2}+\gamma_{k}\left\|G x_{k}-p\right\|^{2} \\
\leqslant & \alpha_{k}\left\|u_{k}-p\right\|^{2}+\beta_{k}\left\|x_{k}-p\right\|^{2}+\gamma_{k}\left\|\tilde{x}_{k}-p\right\|^{2} \\
\leqslant & \alpha_{k}\left\|u_{k}-p\right\|^{2}+\beta_{k}\left\|x_{k}-p\right\|^{2}+\gamma_{k}\left[\left\|x_{k}-p\right\|^{2}-\left\|\left(x_{k}-\tilde{x}_{k}\right)-(p-\tilde{p})\right\|^{2}\right. \\
& \left.+2 v_{2}\left\langle\left(x_{k}-\tilde{x}_{k}\right)-(p-\tilde{p}), F_{2} x_{k}-F_{2} p\right\rangle-v_{2}^{2}\left\|F_{2} x_{k}-F_{2} p\right\|^{2}\right] \\
\leqslant & \alpha_{k}\left\|u_{k}-p\right\|^{2}+\beta_{k}\left\|x_{k}-p\right\|^{2}+\gamma_{k}\left[\left\|x_{k}-p\right\|^{2}-\left\|\left(x_{k}-\tilde{x}_{k}\right)-(p-\tilde{p})\right\|^{2}\right. \\
& \left.+2 v_{2}\left\|\left(x_{k}-\tilde{x}_{k}\right)-(p-\tilde{p})\right\|\left\|F_{2} x_{k}-F_{2} p\right\|\right] \\
\leqslant & \alpha_{k}\left\|u_{k}-p\right\|^{2}+\left(\beta_{k}+\gamma_{k}\right)\left\|x_{k}-p\right\|^{2}-\gamma_{k}\left\|\left(x_{k}-\tilde{x}_{k}\right)-(p-\tilde{p})\right\|^{2} \\
& +2 v_{2}\left\|\left(x_{k}-\tilde{x}_{k}\right)-(p-\tilde{p})\right\|\left\|F_{2} x_{k}-F_{2} p\right\| \\
\leqslant & \alpha_{k}\left\|u_{k}-p\right\|^{2}+\left\|x_{k}-p\right\|^{2}-\gamma_{k}\left\|\left(x_{k}-\tilde{x}_{k}\right)-(p-\tilde{p})\right\|^{2}+2 v_{2}\left\|\left(x_{k}-\tilde{x}_{k}\right)-(p-\tilde{p})\right\|\left\|F_{2} x_{k}-F_{2} p\right\|,
\end{aligned}
$$

which together with (3.15), leads to

$$
\begin{aligned}
\left\|x_{k+1}-p\right\|^{2} \leqslant & \alpha_{k}\|u-p\|^{2}+\beta_{k}\left\|x_{k}-p\right\|^{2}+\gamma_{k}\left\|v_{k}-p\right\|^{2}+2 \gamma_{k} \bar{\epsilon}_{k}\left\|z_{k}-p\right\|+\gamma_{k} \bar{\epsilon}_{k}^{2} \\
\leqslant & \alpha_{k}\|u-p\|^{2}+\beta_{k}\left\|x_{k}-p\right\|^{2}+\gamma_{k}\left[\alpha_{k}\left\|u_{k}-p\right\|^{2}+\left\|x_{k}-p\right\|^{2}-\gamma_{k}\left\|\left(x_{k}-\tilde{x}_{k}\right)-(p-\tilde{p})\right\|^{2}\right. \\
& \left.+2 v_{2}\left\|\left(x_{k}-\tilde{x}_{k}\right)-(p-\tilde{p})\right\|\left\|F_{2} x_{k}-F_{2} p\right\|\right]+2 \bar{\epsilon}_{k}\left\|z_{k}-p\right\|+\bar{\epsilon}_{k}^{2} \\
\leqslant & \alpha_{k}\|u-p\|^{2}+\alpha_{k}\left\|u_{k}-p\right\|^{2}+\left(\beta_{k}+\gamma_{k}\right)\left\|x_{k}-p\right\|^{2}-\gamma_{k}^{2}\left\|\left(x_{k}-\tilde{x}_{k}\right)-(p-\tilde{p})\right\|^{2} \\
& +2 v_{2}\left\|\left(x_{k}-\tilde{x}_{k}\right)-(p-\tilde{p})\right\|\left\|F_{2} x_{k}-F_{2} p\right\|+2 \bar{\epsilon}_{k}\left\|z_{k}-p\right\|+\bar{\epsilon}_{k}^{2} \\
\leqslant & \alpha_{k}\|u-p\|^{2}+\alpha_{k}\left\|u_{k}-p\right\|^{2}+\left\|x_{k}-p\right\|^{2}-\gamma_{k}^{2}\left\|\left(x_{k}-\tilde{x}_{k}\right)-(p-\tilde{p})\right\|^{2} \\
& +2 v_{2}\left\|\left(x_{k}-\tilde{x}_{k}\right)-(p-\tilde{p})\right\|\left\|F_{2} x_{k}-F_{2} p\right\|+2 \bar{\epsilon}_{k}\left\|z_{k}-p\right\|+\bar{\epsilon}_{k}^{2} .
\end{aligned}
$$


So, it follows that

$$
\begin{aligned}
\gamma_{k}^{2}\left\|\left(x_{k}-\tilde{x}_{k}\right)-(p-\tilde{p})\right\|^{2} \leqslant & \alpha_{k}\|u-p\|^{2}+\alpha_{k}\left\|u_{k}-p\right\|^{2}+\left\|x_{k}-p\right\|^{2}-\left\|x_{k+1}-p\right\|^{2} \\
& +2 v_{2}\left\|\left(x_{k}-\tilde{x}_{k}\right)-(p-\tilde{p})\right\|\left\|F_{2} x_{k}-F_{2} p\right\|+2 \bar{\epsilon}_{k}\left\|z_{k}-p\right\|+\bar{\epsilon}_{k}^{2} \\
\leqslant & \alpha_{k}\|u-p\|^{2}+\alpha_{k}\left\|u_{k}-p\right\|^{2}+\left\|x_{k}-x_{k+1}\right\|\left(\left\|x_{k}-p\right\|+\left\|x_{k+1}-p\right\|\right) \\
& +2 v_{2}\left\|\left(x_{k}-\tilde{x}_{k}\right)-(p-\tilde{p})\right\|\left\|F_{2} x_{k}-F_{2} p\right\|+2 \bar{\epsilon}_{k}\left\|z_{k}-p\right\|+\bar{\epsilon}_{k}^{2} .
\end{aligned}
$$

Since $\alpha_{k}+\beta_{k}+\gamma_{k}=1, \alpha_{k} \rightarrow 0, \beta_{k} \rightarrow \xi \in\left(\zeta, \frac{1}{2}\right], \bar{\epsilon}_{k} \rightarrow 0,\left\|F_{2} x_{k}-F_{2} p\right\| \rightarrow 0$ (due to (3.17)), and $\left\|x_{k+1}-x_{k}\right\| \rightarrow 0$ (due to Lemma 3.8), we deduce from the boundedness of $\left\{x_{k}\right\},\left\{\tilde{x}_{k}\right\},\left\{u_{k}\right\}$, and $\left\{z_{k}\right\}$ that

$$
\lim _{k \rightarrow \infty}\left\|\left(x_{k}-\tilde{x}_{k}\right)-(p-\tilde{p})\right\|=0 .
$$

Also, utilizing (3.16) and (3.19) we obtain

$$
\begin{aligned}
\left\|v_{k}-p\right\|^{2} \leqslant & \alpha_{k}\left\|u_{k}-p\right\|^{2}+\beta_{k}\left\|x_{k}-p\right\|^{2}+\gamma_{k}\left\|G x_{k}-p\right\|^{2} \\
\leqslant & \alpha_{k}\left\|u_{k}-p\right\|^{2}+\beta_{k}\left\|x_{k}-p\right\|^{2}+\gamma_{k}\left[\left\|x_{k}-p\right\|^{2}-\left\|\left(\tilde{x}_{k}-q_{k}\right)+(p-\tilde{p})\right\|^{2}\right. \\
& \left.+2 v_{1}\left\|F_{1} \tilde{x}_{k}-F_{1} \tilde{p}\right\|\left\|\left(\tilde{x}_{k}-q_{k}\right)+(p-\tilde{p})\right\|\right] \\
\leqslant & \alpha_{k}\left\|u_{k}-p\right\|^{2}+\left(\beta_{k}+\gamma_{k}\right)\left\|x_{k}-p\right\|^{2}-\gamma_{k}\left\|\left(\tilde{x}_{k}-q_{k}\right)+(p-\tilde{p})\right\|^{2} \\
& +2 v_{1}\left\|F_{1} \tilde{x}_{k}-F_{1} \tilde{p}\right\|\left\|\left(\tilde{x}_{k}-q_{k}\right)+(p-\tilde{p})\right\| \\
\leqslant & \alpha_{k}\left\|u_{k}-p\right\|^{2}+\left\|x_{k}-p\right\|^{2}-\gamma_{k}\left\|\left(\tilde{x}_{k}-q_{k}\right)+(p-\tilde{p})\right\|^{2}+2 v_{1}\left\|F_{1} \tilde{x}_{k}-F_{1} \tilde{p}\right\|\left\|\left(\tilde{x}_{k}-q_{k}\right)+(p-\tilde{p})\right\|,
\end{aligned}
$$

which together with (3.15), leads to

$$
\begin{aligned}
\left\|x_{k+1}-p\right\|^{2} \leqslant & \alpha_{k}\|u-p\|^{2}+\beta_{k}\left\|x_{k}-p\right\|^{2}+\gamma_{k}\left\|v_{k}-p\right\|^{2}+2 \gamma_{k} \bar{\epsilon}_{k}\left\|z_{k}-p\right\|+\gamma_{k} \bar{\epsilon}_{k}^{2} \\
\leqslant & \alpha_{k}\|u-p\|^{2}+\beta_{k}\left\|x_{k}-p\right\|^{2}+\gamma_{k}\left[\alpha_{k}\left\|u_{k}-p\right\|^{2}+\left\|x_{k}-p\right\|^{2}-\gamma_{k}\left\|\left(\tilde{x}_{k}-q_{k}\right)+(p-\tilde{p})\right\|^{2}\right. \\
& \left.+2 v_{1}\left\|F_{1} \tilde{x}_{k}-F_{1} \tilde{p}\right\|\left\|\left(\tilde{x}_{k}-q_{k}\right)+(p-\tilde{p})\right\|\right]+2 \bar{\epsilon}_{k}\left\|z_{k}-p\right\|+\bar{\epsilon}_{k}^{2} \\
\leqslant & \alpha_{k}\|u-p\|^{2}+\alpha_{k}\left\|u_{k}-p\right\|^{2}+\left(\beta_{k}+\gamma_{k}\right)\left\|x_{k}-p\right\|^{2}-\gamma_{k}^{2}\left\|\left(\tilde{x}_{k}-q_{k}\right)+(p-\tilde{p})\right\|^{2} \\
& +2 v_{1}\left\|F_{1} \tilde{x}_{k}-F_{1} \tilde{p}\right\|\left\|\left(\tilde{x}_{k}-q_{k}\right)+(p-\tilde{p})\right\|+2 \bar{\epsilon}_{k}\left\|z_{k}-p\right\|+\bar{\epsilon}_{k}^{2} \\
\leqslant & \alpha_{k}\|u-p\|^{2}+\alpha_{k}\left\|u_{k}-p\right\|^{2}+\left\|x_{k}-p\right\|^{2}-\gamma_{k}^{2}\left\|\left(\tilde{x}_{k}-q_{k}\right)+(p-\tilde{p})\right\|^{2} \\
& +2 v_{1}\left\|F_{1} \tilde{x}_{k}-F_{1} \tilde{p}\right\|\left\|\left(\tilde{x}_{k}-q_{k}\right)+(p-\tilde{p})\right\|+2 \bar{\epsilon}_{k}\left\|z_{k}-p\right\|+\bar{\epsilon}_{k}^{2} .
\end{aligned}
$$

So, it follows that

$$
\begin{aligned}
\gamma_{k}^{2}\left\|\left(\tilde{x}_{k}-q_{k}\right)+(p-\tilde{p})\right\|^{2} \leqslant & \alpha_{k}\|u-p\|^{2}+\alpha_{k}\left\|u_{k}-p\right\|^{2}+\left\|x_{k}-p\right\|^{2}-\left\|x_{k+1}-p\right\|^{2} \\
& +2 v_{1}\left\|F_{1} \tilde{x}_{k}-F_{1} \tilde{p}\right\|\left\|\left(\tilde{x}_{k}-q_{k}\right)+(p-\tilde{p})\right\|+2 \bar{\epsilon}_{k}\left\|z_{k}-p\right\|+\bar{\epsilon}_{k}^{2} \\
\leqslant & \alpha_{k}\|u-p\|^{2}+\alpha_{k}\left\|u_{k}-p\right\|^{2}+\left\|x_{k}-x_{k+1}\right\|\left(\left\|x_{k}-p\right\|+\left\|x_{k+1}-p\right\|\right) \\
& +2 v_{1}\left\|F_{1} \tilde{x}_{k}-F_{1} \tilde{p}\right\|\left\|\left(\tilde{x}_{k}-q_{k}\right)+(p-\tilde{p})\right\|+2 \bar{\epsilon}_{k}\left\|z_{k}-p\right\|+\bar{\epsilon}_{k}^{2} .
\end{aligned}
$$

Since $\alpha_{k}+\beta_{k}+\gamma_{k}=1, \alpha_{k} \rightarrow 0, \beta_{k} \rightarrow \xi \in\left(\zeta, \frac{1}{2}\right], \bar{\epsilon}_{k} \rightarrow 0,\left\|F_{1} \tilde{x}_{k}-F_{1} \tilde{p}\right\| \rightarrow 0$ (due to (3.17)), and $\left\|x_{k+1}-x_{k}\right\| \rightarrow 0$ (due to Lemma 3.8), we deduce from the boundedness of $\left\{x_{k}\right\},\left\{\tilde{x}_{k}\right\},\left\{u_{k}\right\}$, and $\left\{z_{k}\right\}$ that

$$
\lim _{k \rightarrow \infty}\left\|\left(\tilde{x}_{k}-q_{k}\right)+(p-\tilde{p})\right\|=0 .
$$

Note that

$$
\left\|x_{k}-q_{k}\right\| \leqslant\left\|\left(x_{k}-\tilde{x}_{k}\right)-(p-\tilde{p})\right\|+\left\|\left(\tilde{x}_{k}-q_{k}\right)+(p-\tilde{p})\right\| .
$$

Hence from (3.20) and (3.21) we get

$$
\lim _{k \rightarrow \infty}\left\|x_{k}-G x_{k}\right\|=\lim _{k \rightarrow \infty}\left\|x_{k}-q_{k}\right\|=0 .
$$


Also, observe that

$$
\begin{aligned}
\left\|\tilde{u}_{k}-x_{k}\right\| & \leqslant \mu\left\|G x_{k}-x_{k}\right\|+(1-\mu)\left\|T G x_{k}-x_{k}\right\| \\
& \leqslant \mu\left\|G x_{k}-x_{k}\right\|+(1-\mu)\left(\left\|T G x_{k}-G x_{k}\right\|+\left\|G x_{k}-x_{k}\right\|\right) \\
& =\left\|G x_{k}-x_{k}\right\|+(1-\mu)\left\|T G x_{k}-G x_{k}\right\| \\
& \leqslant\left\|G x_{k}-x_{k}\right\|+\left\|T G x_{k}-G x_{k}\right\|
\end{aligned}
$$

So, from (3.17) and (3.22) we know that

$$
\lim _{k \rightarrow \infty}\left\|\tilde{u}_{k}-x_{k}\right\|=0
$$

Taking into consideration that

$$
\left\|u_{k}-x_{k}\right\| \leqslant \alpha_{k}\left\|f\left(x_{k}\right)-x_{k}\right\|+\left(1-\alpha_{k}\right)\left\|G x_{k}-x_{k}\right\| \leqslant \alpha_{k}\left\|f\left(x_{k}\right)-x_{k}\right\|+\left\|G x_{k}-x_{k}\right\|,
$$

and

$$
\left\|v_{k}-x_{k}\right\| \leqslant \alpha_{k}\left\|u_{k}-x_{k}\right\|+\gamma_{k}\left\|\tilde{u}_{k}-x_{k}\right\| \leqslant \alpha_{k}\left\|u_{k}-x_{k}\right\|+\left\|\tilde{u}_{k}-x_{k}\right\|,
$$

we conclude from (3.17), (3.23), and $\alpha_{k} \rightarrow 0$ that

$$
\lim _{k \rightarrow \infty}\left\|x_{k}-u_{k}\right\|=0 \text { and } \quad \lim _{k \rightarrow \infty}\left\|x_{k}-v_{k}\right\|=0 .
$$

So, it follows from (3.17) that

$$
\lim _{k \rightarrow \infty}\left\|x_{k}-y_{k}\right\|=0 \text { and } \lim _{k \rightarrow \infty}\left\|x_{k}-z_{k}\right\|=0 .
$$

Since $A$ is $\beta$-inverse-strongly monotone, it is known that $A$ is $L_{1}$-Lipschitzian with $L_{1}=1 / \beta$. Again by Proposition 2.2 (iii) and Lemma 3.3 we have

$$
\begin{aligned}
& \left\|P_{\mathrm{VI}(\operatorname{GSVI}(G) \cap \operatorname{Fix}(T), B)}\left(y_{k}-\lambda A y_{k}\right)-x_{k+1}\right\| \\
& \leqslant\left\|P_{V I(G S V I(G) \cap F i x(T), B)}\left(y_{k}-\lambda A y_{k}\right)-P_{V I(G S V I(G) \cap F i x(T), B)}\left(z_{k}-\lambda A z_{k}\right)\right\| \\
& +\left\|P_{\mathrm{VI}(\mathrm{GSVI}(\mathrm{G}) \cap \operatorname{Fix}(\mathrm{T}), \mathrm{B})}\left(z_{\mathrm{k}}-\lambda A z_{\mathrm{k}}\right)-x_{\mathrm{k}+1}\right\| \\
& \leqslant\left(1+\lambda \mathrm{L}_{1}\right)\left\|\mathrm{y}_{\mathrm{k}}-z_{\mathrm{k}}\right\|+\alpha_{\mathrm{k}}\left\|\mathrm{P}_{\mathrm{VI}(\mathrm{GSVI}(\mathrm{G}) \cap \operatorname{Fix}(\mathrm{T}), \mathrm{B})}\left(z_{\mathrm{k}}-\lambda \mathrm{A} z_{\mathrm{k}}\right)-u\right\|
\end{aligned}
$$

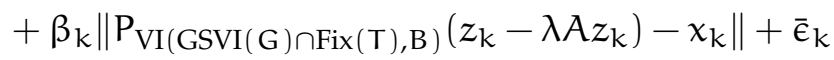

$$
\begin{aligned}
& \leqslant\left(1+\lambda \mathrm{L}_{1}\right)\left\|y_{k}-z_{k}\right\|+\alpha_{k}\left\|P_{\mathrm{VI}(\mathrm{GSVI}(\mathrm{G}) \cap \operatorname{Fix}(\mathrm{T}), \mathrm{B})}\left(z_{\mathrm{k}}-\lambda \mathrm{A} z_{\mathrm{k}}\right)-u\right\|+\bar{\epsilon}_{\mathrm{k}}
\end{aligned}
$$

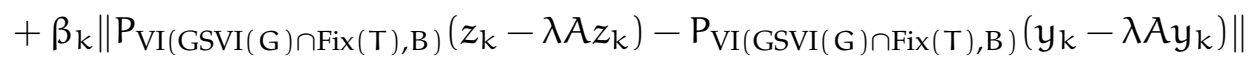

$$
\begin{aligned}
& \left.+\beta_{k} \| P_{V I(G S V I}(G) \cap \operatorname{Fix}(T), B\right)\left(y_{k}-\lambda A y_{k}\right)-y_{k}\left\|+\beta_{k}\right\| y_{k}-x_{k} \| \\
& \leqslant\left(1+\lambda \mathrm{L}_{1}\right)\left\|y_{k}-z_{k}\right\|+\alpha_{k}\left\|P_{\mathrm{VI}(\mathrm{GSVI}(\mathrm{G}) \cap \mathrm{Fix}(\mathrm{T}), \mathrm{B})}\left(z_{\mathrm{k}}-\lambda A z_{\mathrm{k}}\right)-\mathrm{u}\right\|+\bar{\epsilon}_{\mathrm{k}}
\end{aligned}
$$

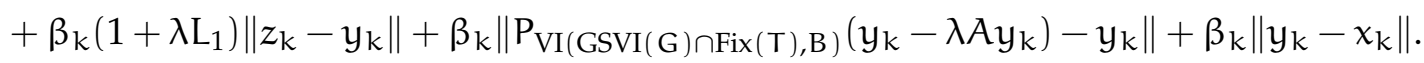

Consequently, from (3.26), we have

$$
\begin{aligned}
& \left\|P_{\mathrm{VI}(\operatorname{GSVI}(G) \cap \operatorname{Fix}(T), B)}\left(y_{k}-\lambda A y_{k}\right)-y_{k}\right\| \\
& \leqslant\left\|P_{\operatorname{VI}(\operatorname{GSVI}(G) \cap \operatorname{Fix}(T), B)}\left(y_{k}-\lambda A y_{k}\right)-x_{k+1}\right\|+\left\|x_{k+1}-x_{k}\right\|+\left\|x_{k}-y_{k}\right\| \\
& \leqslant\left(1+\lambda \mathrm{L}_{1}\right)\left\|y_{k}-z_{k}\right\|+\alpha_{k}\left\|P_{V I(G S V I(G) \cap F i x(T), B)}\left(z_{k}-\lambda A z_{k}\right)-u\right\|+\bar{\epsilon}_{k} \\
& +\beta_{k}\left(1+\lambda L_{1}\right)\left\|z_{k}-y_{k}\right\|+\beta_{k}\left\|P_{V I(G S V I(G) \cap F i x(T), B)}\left(y_{k}-\lambda A y_{k}\right)-y_{k}\right\|+\beta_{k}\left\|y_{k}-x_{k}\right\| \\
& +\left\|x_{k+1}-x_{k}\right\|+\left\|x_{k}-y_{k}\right\|
\end{aligned}
$$

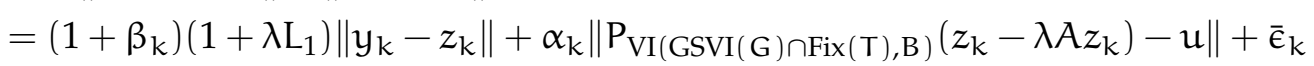

$$
\begin{aligned}
& +\beta_{k}\left\|P_{V I(G S V I(G) \cap F i x(T), B)}\left(y_{k}-\lambda A y_{k}\right)-y_{k}\right\|+\left(1+\beta_{k}\right)\left\|y_{k}-x_{k}\right\|+\left\|x_{k+1}-x_{k}\right\| \text {, }
\end{aligned}
$$


which immediately yields

$$
\begin{aligned}
& \left\|P_{\mathrm{VI}(\operatorname{GSVI}(\mathrm{G}) \cap \operatorname{Fix}(\mathrm{T}), \mathrm{B})}\left(y_{k}-\lambda A y_{k}\right)-y_{k}\right\| \\
& \leqslant \frac{1+\beta_{k}}{1-\beta_{k}}\left(1+\lambda L_{1}\right)\left\|y_{k}-z_{k}\right\|+\frac{\alpha_{k}}{1-\beta_{k}}\left\|P_{V I(G S V I(G) \cap F i x(T), B)}\left(z_{k}-\lambda A z_{k}\right)-u\right\|+\frac{\bar{\epsilon}_{k}}{1-\beta_{k}} \\
& \quad+\frac{1+\beta_{k}}{1-\beta_{k}}\left\|y_{k}-x_{k}\right\|+\frac{1}{1-\beta_{k}}\left\|x_{k+1}-x_{k}\right\| .
\end{aligned}
$$

Since $\alpha_{k}+\beta_{k}+\gamma_{k}=1, \alpha_{k} \rightarrow 0, \beta_{k} \rightarrow \xi \in\left(\zeta, \frac{1}{2}\right], \bar{\epsilon}_{k} \rightarrow 0,\left\|y_{k}-z_{k}\right\| \rightarrow 0,\left\|x_{k}-y_{k}\right\| \rightarrow 0$, and $\left\|x_{k+1}-x_{k}\right\| \rightarrow 0$ (due to Lemma 3.8, (3.17), and (3.25)), we conclude that

$$
\left.\lim _{k \rightarrow \infty} \| P_{V I(G S V I}(G) \cap \operatorname{Fix}(T), B\right)\left(y_{k}-\lambda A y_{k}\right)-y_{k} \|=0 .
$$

From Proposition 2.2 (iii), it follows that

$$
\begin{aligned}
& \left\|\mathrm{P}_{\mathrm{VI}(\mathrm{GSVI}(\mathrm{G}) \cap \operatorname{Fix}(\mathrm{T}), \mathrm{B})}\left(z_{\mathrm{k}}-\lambda \mathrm{A} z_{\mathrm{k}}\right)-z_{\mathrm{k}}\right\| \\
& \left.\leqslant \| P_{V I(G S V I}(G) \cap \operatorname{Fix}(T), B\right)\left(z_{k}-\lambda A z_{k}\right)-P_{V I(G S V I(G) \cap F i x(T), B)}\left(y_{k}-\lambda A y_{k}\right) \| \\
& \left.+\| P_{V I(G S V I}(G) \cap \operatorname{Fix}(T), B\right)\left(y_{k}-\lambda A y_{k}\right)-y_{k}\|+\| y_{k}-z_{k} \| \\
& \leqslant\left(1+\lambda L_{1}\right)\left\|z_{k}-y_{k}\right\|+\left\|P_{V I(G S V I(G) \cap F i x(T), B)}\left(y_{k}-\lambda A y_{k}\right)-y_{k}\right\|+\left\|y_{k}-z_{k}\right\| \\
& \leqslant\left\|P_{V I(G S V I(G) \cap F i x(T), B)}\left(y_{k}-\lambda A y_{k}\right)-y_{k}\right\|+\left(2+\lambda L_{1}\right)\left\|y_{k}-z_{k}\right\| .
\end{aligned}
$$

Utilizing the last inequality we obtain from (3.17) and (3.27) that

$$
\lim _{\mathrm{k} \rightarrow \infty}\left\|\mathrm{P}_{\mathrm{VI}(\mathrm{GSVI}(\mathrm{G}) \cap \operatorname{Fix}(\mathrm{T}), \mathrm{B})}\left(z_{\mathrm{k}}-\lambda \mathrm{A} z_{\mathrm{k}}\right)-z_{\mathrm{k}}\right\|=0 .
$$

Theorem 3.10. Suppose that the hypotheses (H1)-(H4) hold. Then the two sequences $\left\{x_{k}\right\}$ and $\left\{z_{k}\right\}$ in Algorithm 3.2 converge strongly to the same point $x^{*} \in \Omega:=\mathrm{VI}(\mathrm{VI}(\mathrm{GSVI}(\mathrm{G}) \cap \operatorname{Fix}(\mathrm{T}), \mathrm{B}), \mathrm{A})$ provided $\left\|\mathrm{x}_{\mathrm{k}}-v_{\mathrm{k}}\right\|=\mathrm{o}\left(\alpha_{\mathrm{k}}^{2}\right)$, which is a unique solution to the VIP

$$
\left\langle(I-f) x^{*}, p-x^{*}\right\rangle \geqslant 0, \quad \forall p \in \Omega .
$$

Equivalently, $x^{*}=\mathrm{P}_{\Omega} \mathrm{f}\left(\mathrm{x}^{*}\right)$.

Proof. Note that Lemma 3.5 shows the boundedness of $\left\{x_{k}\right\}$. Since $H$ is reflexive, there is at least a weak convergence subsequence of $\left\{x_{k}\right\}$. First, let us assert that $\omega_{w}\left(x_{k}\right) \subset \Omega$. As a matter of fact, take an arbitrary $w \in \omega_{w}\left(x_{k}\right)$. Then there exists a subsequence $\left\{x_{k_{i}}\right\}$ of $\left\{x_{k}\right\}$ such that $x_{k_{i}} \rightarrow w$. From

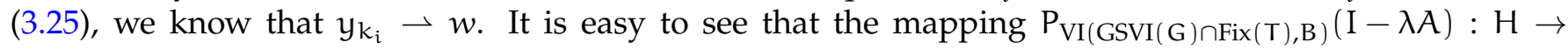
$\operatorname{VI}(G S V I(G) \cap \operatorname{Fix}(T), B) \subset H$ is nonexpansive because $\left.P_{V I(G S V I}(G) \cap F i x(T), B\right)$ is nonexpansive and $I-\lambda A$ is nonexpansive for $\beta$-inverse strongly monotone mapping $A$ with $0<\lambda \leqslant 2 \beta$. So, utilizing Lemma 2.7 and (3.27), we obtain

$$
w=P_{\mathrm{VI}(\mathrm{GSVI}(\mathrm{G}) \cap \mathrm{Fix}(\mathrm{T}), \mathrm{B})}(w-\lambda A w),
$$

which leads to $w \in \operatorname{VI}(\operatorname{VI}(G S V I(G) \cap \operatorname{Fix}(T), B), A)=: \Omega$. Thus, the assertion is valid.

Also, note that

$$
\langle(I-f) x-(I-f) y, x-y\rangle \geqslant(1-\rho)\|x-y\|^{2}, \quad \forall x, y \in H .
$$

Hence, it follows from $0 \leqslant \rho<1$ that $I-f$ is $(1-\rho)$-strongly monotone. In the meantime, it is clear that I $-f$ is Lipschitzian with constant $1+\rho>0$. Thus, by Lemma 2.6 (iv) we know that there exists a unique solution $x^{*} \in \Omega:=\operatorname{VI}(\operatorname{VI}(\operatorname{GSVI}(G) \cap \operatorname{Fix}(T), B), A)$ to the VIP

$$
\left\langle(I-f) x^{*}, p-x^{*}\right\rangle \geqslant 0, \quad \forall p \in \Omega .
$$

Equivalently, $x^{*}=\mathrm{P}_{\Omega} \mathrm{f}\left(\mathrm{x}^{*}\right)$. 
Next, let us show that $x_{k} \rightarrow x^{*}$. Indeed, take an arbitrary $p \in \Omega:=\operatorname{VI}(\operatorname{VI}(\operatorname{GSVI}(G) \cap \operatorname{Fix}(T), B), A)$. Then, from Algorithm 3.2, Lemma 2.5, (3.5), and (3.6), we have

$$
\begin{aligned}
\left\|u_{k}-p\right\|^{2} & =\left\|\alpha_{k}\left(f\left(x_{k}\right)-f(p)\right)+\left(1-\alpha_{k}\right)\left(G x_{k}-p\right)+\alpha_{k}(f(p)-p)\right\|^{2} \\
& \leqslant\left\|\alpha_{k}\left(f\left(x_{k}\right)-f(p)\right)\right\|^{2}+\left(1-\alpha_{k}\right)\left(G x_{k}-p\right) \|^{2}+2 \alpha_{k}\left\langle f(p)-p, u_{k}-p\right\rangle \\
& \leqslant \alpha_{k}\left\|f\left(x_{k}\right)-f(p)\right\|^{2}+\left(1-\alpha_{k}\right)\left\|G x_{k}-p\right\|^{2}+2 \alpha_{k}\left\langle f(p)-p, u_{k}-p\right\rangle \\
& \leqslant \alpha_{k} \rho\left\|x_{k}-p\right\|^{2}+\left(1-\alpha_{k}\right)\left\|x_{k}-p\right\|^{2}+2 \alpha_{k}\left\langle f(p)-p, u_{k}-p\right\rangle \\
& =\left(1-\alpha_{k}(1-\rho)\right)\left\|x_{k}-p\right\|^{2}+2 \alpha_{k}\left\langle f(p)-p, u_{k}-p\right\rangle,
\end{aligned}
$$

and hence

$$
\begin{aligned}
\left\|v_{k}-p\right\|^{2} & =\left\|\alpha_{k} u_{k}+\beta_{k} x_{k}+\gamma_{k} \tilde{u}_{k}-p\right\|^{2} \\
& \leqslant \alpha_{k}\left\|u_{k}-p\right\|^{2}+\beta_{k}\left\|x_{k}-p\right\|^{2}+\gamma_{k}\left\|\tilde{u}_{k}-p\right\|^{2} \\
& \leqslant \alpha_{k}\left[\left(1-\alpha_{k}(1-\rho)\right)\left\|x_{k}-p\right\|^{2}+2 \alpha_{k}\left\langle f(p)-p, u_{k}-p\right\rangle\right]+\beta_{k}\left\|x_{k}-p\right\|^{2}+\gamma_{k}\left\|G x_{k}-p\right\|^{2} \\
& \leqslant \alpha_{k}\left[\left(1-\alpha_{k}(1-\rho)\right)\left\|x_{k}-p\right\|^{2}+2 \alpha_{k}\left\langle f(p)-p, u_{k}-p\right\rangle\right]+\beta_{k}\left\|x_{k}-p\right\|^{2}+\gamma_{k}\left\|x_{k}-p\right\|^{2} \\
& =\left(1-\alpha_{k}^{2}(1-\rho)\right)\left\|x_{k}-p\right\|^{2}+2 \alpha_{k}^{2}\left\langle f(p)-p, u_{k}-p\right\rangle \\
& \leqslant\left\|x_{k}-p\right\|^{2}+2 \alpha_{k}^{2}\left\langle f(p)-p, u_{k}-p\right\rangle,
\end{aligned}
$$

which immediately leads to

$$
\begin{aligned}
0 & \leqslant\left\|x_{k}-p\right\|^{2}-\left\|v_{k}-p\right\|^{2}+2 \alpha_{k}^{2}\left\langle f(p)-p, u_{k}-p\right\rangle \\
& \leqslant\left\|x_{k}-v_{k}\right\|\left(\left\|x_{k}-p\right\|+\left\|v_{k}-p\right\|\right)+2 \alpha_{k}^{2}\left(\left\langle f(p)-p, u_{k}-x_{k}\right\rangle+\left\langle f(p)-p, x_{k}-p\right\rangle\right) \\
& \leqslant\left\|x_{k}-v_{k}\right\|\left(\left\|x_{k}-p\right\|+\left\|v_{k}-p\right\|\right)+2 \alpha_{k}^{2}\left(\|f(p)-p\|\left\|u_{k}-x_{k}\right\|+\left\langle f(p)-p, x_{k}-p\right\rangle\right) .
\end{aligned}
$$

That is,

$$
0 \leqslant \frac{\left\|x_{k}-v_{k}\right\|}{2 \alpha_{k}^{2}}\left(\left\|x_{k}-p\right\|+\left\|v_{k}-p\right\|\right)+\|f(p)-p\|\left\|u_{k}-x_{k}\right\|+\left\langle f(p)-p, x_{k}-p\right\rangle .
$$

Since for any $w \in \omega_{w}\left(x_{k}\right)$ there exists a subsequence $\left\{x_{k_{i}}\right\}$ of $\left\{x_{k}\right\}$ such that $x_{k_{i}} \rightarrow w$, we deduce from (3.24), $\alpha_{k} \rightarrow 0$, and $\left\|x_{k}-v_{k}\right\|=o\left(\alpha_{k}^{2}\right)$ that for all $p \in \Omega:=\operatorname{VI}(\operatorname{VI}(\operatorname{GSVI}(G) \cap \operatorname{Fix}(T), B), A)$

$$
\begin{aligned}
0 & \leqslant \lim _{i \rightarrow \infty}\left\{\frac{\left\|x_{k_{i}}-v_{k_{i}}\right\|}{2 \alpha_{k_{i}}^{2}}\left(\left\|x_{k_{i}}-p\right\|+\left\|v_{k_{i}}-p\right\|\right)+\|f(p)-p\|\left\|u_{k_{i}}-x_{k_{i}}\right\|+\left\langle f(p)-p, x_{k_{i}}-p\right\rangle\right\} \\
& =\lim _{i \rightarrow \infty}\left\langle f(p)-p, x_{k_{i}}-p\right\rangle=\langle(f-I) p, w-p\rangle .
\end{aligned}
$$

That is,

$$
\langle(I-f) p, p-w\rangle \geqslant 0, \quad \forall p \in \Omega .
$$

Consequently, by Lemma 2.6 (i) (Minty's lemma), we know that

$$
\langle(\mathrm{I}-\mathrm{f}) w, \mathrm{p}-w\rangle \geqslant 0, \quad \forall \mathrm{p} \in \Omega,
$$

that is, $w$ is a solution of VIP (3.28). By the uniqueness of solutions of VIP (3.28), we get $w=x^{*}$, which hence implies that $\omega_{w}\left(x_{k}\right)=\left\{x^{*}\right\}$. Therefore, it is known that $\left\{x_{k}\right\}$ converges weakly to the unique solution $x^{*} \in \Omega:=\operatorname{VI}(\operatorname{VI}(\mathrm{GSVI}(\mathrm{G}) \cap \operatorname{Fix}(\mathrm{T}), \mathrm{B}), \mathrm{A})$ of $\operatorname{VIP}(3.28)$.

Finally, let us show that $\left\|x_{k}-x^{*}\right\| \rightarrow 0$ as $k \rightarrow \infty$. Indeed, in terms of Algorithm 3.2 and Lemma 2.5, we conclude from (3.4) and the $\beta$-inverse-strong monotonicity of $A$ with $0<\lambda \leqslant 2 \beta$, that

$$
\begin{aligned}
\left\|x_{k+1}-x^{*}\right\|^{2} & =\left\|\alpha_{k} u+\beta_{k} x_{k}+\gamma_{k} h_{k}-x^{*}\right\|^{2} \\
& \leqslant\left\|\beta_{k}\left(x_{k}-x^{*}\right)+\gamma_{k}\left(h_{k}-x^{*}\right)\right\|^{2}+2 \alpha_{k}\left\langle u-x^{*}, x_{k+1}-x^{*}\right\rangle
\end{aligned}
$$




$$
\begin{aligned}
& \leqslant \beta_{\mathrm{k}}\left\|x_{\mathrm{k}}-x^{*}\right\|^{2}+\gamma_{\mathrm{k}}\left\|\mathrm{h}_{\mathrm{k}}-x^{*}\right\|^{2}+2 \alpha_{\mathrm{k}}\left\langle\mathrm{u}-\mathrm{x}^{*}, \mathrm{x}_{\mathrm{k}+1}-x^{*}\right\rangle
\end{aligned}
$$

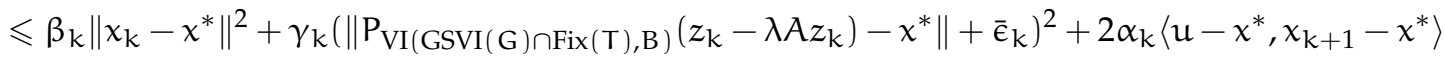

$$
\begin{aligned}
& =\beta_{k}\left\|x_{k}-x^{*}\right\|^{2}+\gamma_{k}\left(\| P_{V I(G S V I}(G) \cap \operatorname{Fix}(T), B\right)\left(z_{k}-\lambda A z_{k}\right) \\
& \left.-\mathrm{P}_{\mathrm{VI}(\mathrm{GSVI}(\mathrm{G}) \cap \mathrm{Fix}(\mathrm{T}), \mathrm{B})}\left(\mathrm{x}^{*}-\lambda A x^{*}\right) \|+\bar{\epsilon}_{\mathrm{k}}\right)^{2}+2 \alpha_{\mathrm{k}}\left\langle\mathrm{u}-\mathrm{x}^{*}, \mathrm{x}_{\mathrm{k}+1}-\mathrm{x}^{*}\right\rangle \\
& \leqslant \beta_{k}\left\|x_{k}-x^{*}\right\|^{2}+\gamma_{k}\left(\left\|(I-\lambda A) z_{k}-(I-\lambda A) x^{*}\right\|+\bar{\epsilon}_{k}\right)^{2}+2 \alpha_{k}\left\langle u-x^{*}, x_{k+1}-x^{*}\right\rangle \\
& \leqslant \beta_{\mathrm{k}}\left\|x_{\mathrm{k}}-x^{*}\right\|^{2}+\gamma_{\mathrm{k}}\left(\left\|z_{\mathrm{k}}-\mathrm{x}^{*}\right\|+\bar{\epsilon}_{\mathrm{k}}\right)^{2}+2 \alpha_{\mathrm{k}}\left\langle\mathrm{u}-\mathrm{x}^{*}, \mathrm{x}_{\mathrm{k}+1}-\mathrm{x}^{*}\right\rangle \\
& =\beta_{k}\left\|x_{k}-x^{*}\right\|^{2}+\gamma_{k}\left\|z_{k}-x^{*}\right\|^{2}+\gamma_{k} \bar{\epsilon}_{k}\left(2\left\|z_{k}-x^{*}\right\|+\bar{\epsilon}_{k}\right)+2 \alpha_{k}\left\langle u-x^{*}, x_{k+1}-x^{*}\right\rangle \\
& \leqslant \beta_{k}\left\|x_{k}-x^{*}\right\|^{2}+\gamma_{k}\left\|v_{k}-x^{*}\right\|^{2}+\gamma_{k} \bar{\epsilon}_{k}\left(2\left\|z_{k}-x^{*}\right\|+\bar{\epsilon}_{k}\right)+2 \alpha_{k}\left\langle u-x^{*}, x_{k+1}-x^{*}\right\rangle \\
& =\beta_{k}\left\|x_{k}-x^{*}\right\|^{2}+\gamma_{k}\left\|x_{k}-x^{*}+v_{k}-x_{k}\right\|^{2}+\gamma_{k} \bar{\epsilon}_{k}\left(2\left\|z_{k}-x^{*}\right\|+\bar{\epsilon}_{k}\right)+2 \alpha_{k}\left\langle u-x^{*}, x_{k+1}-x^{*}\right\rangle \\
& =\beta_{k}\left\|x_{k}-x^{*}\right\|^{2}+\gamma_{k}\left(\left\|x_{k}-x^{*}\right\|^{2}+2\left\langle x_{k}-x^{*}, v_{k}-x_{k}\right\rangle+\left\|v_{k}-x_{k}\right\|^{2}\right) \\
& +\gamma_{k} \bar{\epsilon}_{k}\left(2\left\|z_{k}-x^{*}\right\|+\bar{\epsilon}_{k}\right)+2 \alpha_{k}\left\langle u-x^{*}, x_{k+1}-x^{*}\right\rangle \text {. }
\end{aligned}
$$

It follows that

$$
\begin{aligned}
\left\|x_{k+1}-x^{*}\right\|^{2} \leqslant & \beta_{k}\left\|x_{k}-x^{*}\right\|^{2}+\gamma_{k}\left(\left\|x_{k}-x^{*}\right\|^{2}+\left\|v_{k}-x_{k}\right\|\left(2\left\|x_{k}-x^{*}\right\|+\left\|v_{k}-x_{k}\right\|\right)\right. \\
& +\gamma_{k} \bar{\epsilon}_{k}\left(2\left\|z_{k}-x^{*}\right\|+\bar{\epsilon}_{k}\right)+2 \alpha_{k}\left\langle u-x^{*}, x_{k+1}-x^{*}\right\rangle \\
\leqslant & \left(1-\alpha_{k}\right)\left\|x_{k}-x^{*}\right\|^{2}+\left\|v_{k}-x_{k}\right\|\left(2\left\|x_{k}-x^{*}\right\|+\left\|v_{k}-x_{k}\right\|\right) \\
& +\bar{\epsilon}_{k}\left(2\left\|z_{k}-x^{*}\right\|+\bar{\epsilon}_{k}\right)+2 \alpha_{k}\left\langle u-x^{*}, x_{k+1}-x^{*}\right\rangle \\
= & \left(1-\alpha_{k}\right)\left\|x_{k}-x^{*}\right\|^{2}+\alpha_{k}\left[\frac{\left\|v_{k}-x_{k}\right\|}{\alpha_{k}}\left(2\left\|x_{k}-x^{*}\right\|+\left\|v_{k}-x_{k}\right\|\right)\right. \\
& \left.+2\left\langle u-x^{*}, x_{k+1}-x^{*}\right\rangle\right]+\bar{\epsilon}_{k}\left(2\left\|z_{k}-x^{*}\right\|+\bar{\epsilon}_{k}\right) .
\end{aligned}
$$

Since $\alpha_{k} \rightarrow 0,\left\|x_{k}-v_{k}\right\|=o\left(\alpha_{k}\right), \sum_{k=0}^{\infty} \bar{\epsilon}_{k}<\infty$, and $x_{k} \rightarrow x^{*}$, we deduce from the boundedness of $\left\{x_{k}\right\},\left\{v_{k}\right\},\left\{z_{k}\right\}$ that $\sum_{k=0}^{\infty} \bar{\epsilon}_{k}\left(2\left\|z_{k}-x^{*}\right\|+\bar{\epsilon}_{k}\right)<\infty$ and

$$
\limsup _{k \rightarrow \infty}\left[\frac{\left\|v_{k}-x_{k}\right\|}{\alpha_{k}}\left(2\left\|x_{k}-x^{*}\right\|+\left\|v_{k}-x_{k}\right\|\right)+2\left\langle u-x^{*}, x_{k+1}-x^{*}\right\rangle\right] \leqslant 0 .
$$

Therefore, applying Lemma 2.12 to (3.29), we infer from $\sum_{k=0}^{\infty} \alpha_{k}=\infty$ that $\left\|x_{k}-x^{*}\right\| \rightarrow 0$ as $k \rightarrow \infty$. Utilizing (3.25) we also obtain that $\left\|z_{k}-x^{*}\right\| \rightarrow 0$ as $k \rightarrow \infty$. This completes the proof.

Theorem 3.11. Suppose that the hypotheses (H1)-(H4) hold. Then the two sequences $\left\{x_{k}\right\}$ and $\left\{z_{k}\right\}$ in Algorithm 3.2 converge strongly to the same point $x^{*} \in \Omega:=\mathrm{VI}(\mathrm{VI}(\mathrm{GSVI}(\mathrm{G}) \cap \operatorname{Fix}(\mathrm{T}), \mathrm{B}), \mathrm{A})$, where $\mathrm{x}^{*}=\mathrm{P}_{\Omega} \mathrm{u}$, i.e., $\left\|\mathrm{u}-x^{*}\right\|=\inf _{\mathfrak{p} \in \Omega}\|\mathrm{u}-\mathrm{p}\|$.

Proof. Assume that the hypotheses (H1)-(H4) hold and that $\left\|x_{k+1}-x_{k}\right\|=o\left(\alpha_{k}^{2}\right)$. In this case, it is easy to see that Lemmas 3.3-3.6, 3.8 and 3.9 hold.

Next, we divide the rest of the proof into several steps.

Step 1. Repeating the same arguments as those of (3.24) and (3.25), we can prove that

$$
\lim _{k \rightarrow \infty}\left\|x_{k}-u_{k}\right\|=0, \quad \lim _{k \rightarrow \infty}\left\|x_{k}-v_{k}\right\|=0, \quad \lim _{k \rightarrow \infty}\left\|x_{k}-y_{k}\right\|=0, \quad \lim _{k \rightarrow \infty}\left\|x_{k}-z_{k}\right\|=0 .
$$

Step 2. We prove that $\omega_{w}\left(x_{k}\right) \subset \Omega:=\operatorname{VI}(\operatorname{VI}(G S V I(G) \cap \operatorname{Fix}(T), B), A)$.

Indeed, from Lemma 3.9 and $\lim _{k \rightarrow \infty}\left\|x_{k}-v_{k}\right\|=0$, we have

$$
\begin{aligned}
& \lim _{k \rightarrow \infty}\left\|\operatorname{PVI}_{\operatorname{ViSVI}(G) \cap \operatorname{Fix}(\mathrm{T}), \mathrm{B})}\left(z_{k}-\lambda_{k} A z_{k}\right)-z_{k}\right\|=0, \\
& \lim _{k \rightarrow \infty}\left\|\operatorname{PVI}_{\operatorname{VI}(G V I(G) \cap \operatorname{Fix}(T), B)}\left(y_{k}-\lambda_{k} A y_{k}\right)-y_{k}\right\|=0 .
\end{aligned}
$$

Utilizing the same argument as in the proof of Theorem 3.10, we obtain that $\omega_{w}\left(x_{k}\right) \subset \Omega$. 
Step 3. We prove that $\lim _{k \rightarrow \infty}\left\|x_{k}-x^{*}\right\|=0$ where $x^{*}=P_{\Omega} u$.

Indeed, we may assume, without loss of generality, that there exists a subsequence $\left\{x_{k_{i}}\right\}$ of $\left\{x_{k}\right\}$ such that

$$
\limsup _{k \rightarrow \infty}\left\langle u-x^{*}, x_{k}-x^{*}\right\rangle=\lim _{i \rightarrow \infty}\left\langle u-x^{*}, x_{k_{i}}-x^{*}\right\rangle
$$

and $x_{k_{i}} \rightarrow w \in \Omega$. Since $x^{*}=P_{\Omega} u$ and $\left\|x_{k+1}-x_{k}\right\| \rightarrow 0$, we have

$$
\limsup _{k \rightarrow \infty}\left\langle u-x^{*}, x_{k+1}-x^{*}\right\rangle=\lim _{i \rightarrow \infty}\left\langle u-x^{*}, x_{k_{i}}-x^{*}\right\rangle=\left\langle u-x^{*}, w-x^{*}\right\rangle \leqslant 0 .
$$

Utilizing the similar arguments to those of (3.29) and (3.30), we get

$$
\left\|v_{k}-x^{*}\right\|^{2} \leqslant\left\|x_{k}-x^{*}\right\|^{2}+2 \alpha_{k}^{2}\left\langle f\left(x^{*}\right)-x^{*}, u_{k}-x^{*}\right\rangle,
$$

and

$$
\left\|x_{k+1}-x^{*}\right\|^{2} \leqslant \beta_{k}\left\|x_{k}-x^{*}\right\|^{2}+\gamma_{k}\left\|\nu_{k}-x^{*}\right\|^{2}+\gamma_{k} \bar{\epsilon}_{k}\left(2\left\|z_{k}-x^{*}\right\|+\bar{\epsilon}_{k}\right)+2 \alpha_{k}\left\langle u-x^{*}, x_{k+1}-x^{*}\right\rangle .
$$

Combining the last two inequalities, we get

$$
\begin{aligned}
\left\|x_{k+1}-x^{*}\right\|^{2} \leqslant & \beta_{k}\left\|x_{k}-x^{*}\right\|^{2}+\gamma_{k}\left[\left\|x_{k}-x^{*}\right\|^{2}+2 \alpha_{k}^{2}\left\langle f\left(x^{*}\right)-x^{*}, u_{k}-x^{*}\right\rangle\right] \\
& +\gamma_{k} \bar{\epsilon}_{k}\left(2\left\|z_{k}-x^{*}\right\|+\bar{\epsilon}_{k}\right)+2 \alpha_{k}\left\langle u-x^{*}, x_{k+1}-x^{*}\right\rangle \\
= & \left(\beta_{k}+\gamma_{k}\right)\left\|x_{k}-x^{*}\right\|^{2}+2 \gamma_{k} \alpha_{k}^{2}\left\langle f\left(x^{*}\right)-x^{*}, u_{k}-x^{*}\right\rangle \\
& +\gamma_{k} \bar{\epsilon}_{k}\left(2\left\|z_{k}-x^{*}\right\|+\bar{\epsilon}_{k}\right)+2 \alpha_{k}\left\langle u-x^{*}, x_{k+1}-x^{*}\right\rangle \\
\leqslant & \left(1-\alpha_{k}\right)\left\|x_{k}-x^{*}\right\|^{2}+2 \alpha_{k}^{2}\left\|f\left(x^{*}\right)-x^{*}\right\|\left\|u_{k}-x^{*}\right\| \\
& +\bar{\epsilon}_{k}\left(2\left\|z_{k}-x^{*}\right\|+\bar{\epsilon}_{k}\right)+2 \alpha_{k}\left\langle u-x^{*}, x_{k+1}-x^{*}\right\rangle \\
= & \left(1-\alpha_{k}\right)\left\|x_{k}-x^{*}\right\|^{2}+\alpha_{k} \cdot 2\left(\alpha_{k}\left\|f\left(x^{*}\right)-x^{*}\right\|\left\|u_{k}-x^{*}\right\|\right. \\
& \left.+\left\langle u-x^{*}, x_{k+1}-x^{*}\right\rangle\right)+\bar{\epsilon}_{k}\left(2\left\|z_{k}-x^{*}\right\|+\bar{\epsilon}_{k}\right) \\
= & \left(1-s_{k}\right)\left\|x_{k}-x^{*}\right\|^{2}+s_{k} \cdot t_{k}+r_{k},
\end{aligned}
$$

where $s_{k}=\alpha_{k}, t_{k}=2\left(\alpha_{k}\left\|f\left(x^{*}\right)-x^{*}\right\|\left\|u_{k}-x^{*}\right\|+\left\langle u-x^{*}, x_{k+1}-x^{*}\right\rangle\right)$ and $r_{k}=\bar{\epsilon}_{k}\left(2\left\|z_{k}-x^{*}\right\|+\bar{\epsilon}_{k}\right)$. Since $\alpha_{k} \rightarrow 0, \sum_{k=0}^{\infty} \alpha_{k}=\infty, \sum_{k=0}^{\infty} \bar{\epsilon}_{k}<\infty$, and $\lim \sup _{k \rightarrow \infty}\left\langle u-x^{*}, x_{k+1}-x^{*}\right\rangle \leqslant 0$ (due to (3.31)), we deduce from the boundedness of $\left\{x_{k}\right\},\left\{u_{k}\right\},\left\{z_{k}\right\}$ that $\limsup _{k \rightarrow \infty} t_{k} \leqslant 0, \sum_{k=0}^{\infty} s_{k}=\infty$, and $\sum_{k=0}^{\infty} r_{k}<\infty$. Therefore, applying Lemma 2.12 to (3.32), we obtain

$$
\lim _{k \rightarrow \infty}\left\|x_{k}-x^{*}\right\|=0
$$

This completes the proof.

\section{Acknowledgment}

This work was supported by the Innovation Program of Shanghai Municipal Education Commission (15ZZ068), Ph.D. Program Foundation of Ministry of Education of China (20123127110002) and Program for Shanghai Outstanding Academic Leaders in Shanghai City (15XD1503100). This study was supported by the grant from Research Center for Nonlinear Analysis and Optimization of Kaohsiung Medical University. Yeong-Cheng Liou was supported in part by the grand form Kaohsiung Medical University Research Foundation (KMU-Q106005) and Taiwan-Russian joint grant MOST 106-2923-E-039-001-MY3.

\section{References}

[1] P. N. Anh, J. K. Kim, L. D. Muu, An extragradient algorithm for solving bilevel pseudomonotone variational inequalities, J. Global Optim., 52 (2012), 627-639. 1, 1.2, 1

[2] L.-C. Ceng, Q. H. Ansari, M. M. Wong, J.-C. Yao, Mann type hybrid extragradient method for variational inequalities, variational inclusions and fixed point problems, Fixed Point Theory, 13 (2012), 403-422. 1, 1

[3] L.-C. Ceng, Q. H. Ansari, J.-C. Yao, Relaxed extragradient iterative methods for variational inequalities, Appl. Math. Comput., 218 (2011), 1112-1123.1, 1 
[4] L.-C. Ceng, Q. H. Ansari, J.-C. Yao, Relaxed hybrid steepest-descent methods with variable parameters for triplehierarchical variational inequalities, Appl. Anal., 91 (2012), 1793-1810. 2.14

[5] L.-C. Ceng, S.-M. Guu, J.-C. Yao, A general composite iterative algorithm for nonexpansive mappings in Hilbert spaces, Comput. Math. Appl., 61 (2011), 2447-2455. 1

[6] L.-C. Ceng, C.-T. Pang, C.-F. Wen, Multi-step extragradient method with regularization for triple hierarchical variational inequalities with variational inclusion and split feasibility constraints, J. Inequal. Appl., 2014 (2014), 40 pages. 1, 1

[7] L.-C. Ceng, C.-Y. Wang, J.-C. Yao, Strong convergence theorems by a relaxed extragradient method for a general system of variational inequalities, Math. Methods Oper. Res., 67 (2008), 375-390. 1, 2.11

[8] L.-C. Ceng, J.-C. Yao, A relaxed extragradient-like method for a generalized mixed equilibrium problem, a general system of generalized equilibria and a fixed point problem, Nonlinear Anal., 72 (2010), 1922-1937. 1

[9] S. Y. Cho, B. A. Bin Dehaish, X.-L. Qin, Weak convergence of a splitting algorithm in Hilbert spaces, J. Appl. Anal. Comput., 7 (2017), 427-437. 1

[10] N.-N. Fang, Y.-P. Gong, Viscosity iterative methods for split variational inclusion problems and fixed point problems of a nonexpansive mapping, Commun. Optim. Theory, 2016 (2016), 15 pages. 1

[11] K. Goebel, W. A. Kirk, Topics in metric fixed point theory, Cambridge Studies in Advanced Mathematics, Cambridge University Press, Cambridge, (1990). 2.7, 2.13

[12] K. Geobel, S. Reich, Uniform convexity, hyperbolic geometry, and nonexpansive mappings, Monographs and Textbooks in Pure and Applied Mathematics, Marcel Dekker, Inc., New York, (1984). 2.4

[13] H. Iiduka, Strong convergence for an iterative method for the triple-hierarchical constrained optimization problem, Nonlinear Anal., 71 (2009), 1292-1297. 1

[14] H. Iiduka, Iterative algorithm for solving triple-hierarchical constrained optimization problem, J. Optim. Theory Appl., 148 (2011), 580-592. 1, 1.3, 1.4, 1

[15] D. Kinderlehrer, G. Stampacchia, An introduction to variational inequalities and their applications, Pure and Applied Mathematics, Academic Press, Inc. [Harcourt Brace Jovanovich, Publishers], New York-London, (1980). 2.6

[16] G. M. Korpelević, An extragradient method for finding saddle points and for other problems, (Russian) Ékonom. i Mat. Metody, 12 (1976), 747-756. 1, 1

[17] L. S. Liu, Ishikawa and Mann iterative process with errors for nonlinear strongly accretive mappings in Banach spaces, J. Math. Anal. Appl., 194 (1995), 114-125. 2.12

[18] Z.-Q. Luo, J.-S. Pang, D. Ralph, Mathematical programs with equilibrium constraints, Cambridge University Press, Cambridge, (1996). 1, 1

[19] G. Marino, H.-K. Xu, Weak and strong convergence theorems for strict pseudo-contractions in Hilbert spaces, J. Math. Anal. Appl., 329 (2007), 336-346. 2.8

[20] J. Outrata, M. Kočvara, J. Zowe, Nonsmooth approach to optimization problems with equilibrium constraints, Theory, applications and numerical results, Nonconvex Optimization and its Applications, Kluwer Academic Publishers, Dordrecht, (1998). 1

[21] H. Piri, R. Yavarimehr, Solving systems of monotone variational inequalities on fixed point sets of strictly pseudocontractive mappings, J. Nonlinear Funct. Anal., 2016 (2016), 18 pages. 1

[22] X.-L. Qin, S. Y. Cho, Convergence analysis of a monotone projection algorithm in reflexive Banach spaces, Acta Math. Sci. Ser. B Engl. Ed., 37 (2017), 488-502. 1

[23] M. Solodov, An explicit descent method for bilevel convex optimization, J. Convex Anal., 14 (2007), 227-237. 1

[24] V. V. Vasin, A. L. Ageev, Ill-posed problems with a priori information, Inverse and Ill-posed Problems Series. VSP, Utrecht, (1995). 2.6

[25] R. U. Verma, On a new system of nonlinear variational inequalities and associated iterative algorithms, Math. Sci. Res. Hot-Line, 3 (1999), 65-68. 1

[26] Y.-H. Yao, R.-D. Chen, H.-K. Xu, Schemes for finding minimum-norm solutions of variational inequalities, Nonlinear Anal., 72 (2010), 3447-3456. 2.2

[27] Y.-H. Yao, Y.-C. Liou, S. M. Kang, Approach to common elements of variational inequality problems and fixed point problems via a relaxed extragradient method, Comput. Math. Appl., 59 (2010), 3472-3480. 1, 2.9

[28] Y.-H. Yao, Y.-C. Liou, J.-C. Yao, An extragradient method for fixed point problems and variational inequality problems, J. Inequal. Appl., 2007 (2007), 12 pages. 3

[29] Y.-H. Yao, M. A. Noor, Y.-C. Liou, Strong convergence of a modified extragradient method to the minimum-norm solution of variational inequalities, Abstr. Appl. Anal., 2012 (2012), 9 pages. 1

[30] Y.-H. Yao, M. A. Noor, Y.-C. Liou, S. M. Kang, Iterative algorithms for general multivalued variational inequalities, Abstr. Appl. Anal., 2012 (2012), 10 pages. 1

[31] Y.-H. Yao, M. Postolache, Y.-C. Liou, Z.-S. Yao, Construction algorithms for a class of monotone variational inequalities, Optim. Lett., 10 (2016), 1519-1528. 1

[32] H. Zegeye, N. Shahzad, Y.-H. Yao, Minimum-norm solution of variational inequality and fixed point problem in Banach spaces, Optimization, 64 (2015), 453-471. 1

[33] L.-C. Zeng, M. M. Wong, J.-C. Yao, Strong convergence of relaxed hybrid steepest-descent methods for triple hierarchical constrained optimization, Fixed Point Theory Appl., 2012 (2012), 24 pages. 1, 1 